\title{
Projectivity of Hopf algebras over subalgebras with semilocal central localizations
}

\author{
Serge Skryabin \\ Chebotarev Research Institute, Universitetskaya St. 17, 420008 Kazan, Russia \\ E-mail: Serge.Skryabin@ksu.ru
}

\section{Introduction}

Let $H$ be a Hopf algebra over a field $k$ and $A$ a right coideal subalgebra of $H$, that is, $A$ is a subalgebra satisfying $\Delta(A) \subset A \otimes H$ where $\Delta$ is the comultiplication in $H$. In case when $H$ is finitely generated commutative, the right coideal subalgebras are intimately related to the homogeneous spaces for the corresponding group scheme. The purpose of this paper is to extend the class of pairs $A, H$ for which $H$ is proved to be either projective or flat as a module over $A$. As is known the faithful flatness over Hopf subalgebras may be lacking in general. Examples given by Schauenburg [26] use some extremely big Hopf algebras coming from a universal construction of [31]. Positive results can be expected therefore only under some finiteness assumptions.

A Hopf algebra is called residually finite dimensional [19] if its ideals of finite codimension have zero intersection. Many important classes of Hopf algebras are residually finite dimensional. Among them are the finitely generated commutative Hopf algebras, the universal enveloping algebras of finite dimensional Lie algebras, and also Hopf algebras related to quantum groups.

We say that a ring $R$ has semilocal localizations with respect to a central subring $Z$ if for each maximal ideal $\mathfrak{m}$ of $Z$ the localization $R_{\mathfrak{m}}$ of $R$ at the multiplicatively closed set $Z \backslash \mathfrak{m}$ is a semilocal ring whose Jacobson radical contains $\mathfrak{m} R_{\mathfrak{m}}$. For instance, this property is satisfied for any ring module-finite over a central subring. For each ring $R$ let $\mathcal{M}_{R}$ and ${ }_{R} \mathcal{M}$ denote the categories of right and left $R$-modules, respectively.

Theorem 0.1. Let $H$ be a residually finite dimensional Hopf algebra, and let $A$ be a Hopf subalgebra having semilocal localizations with respect to a central subring $Z$. Then $H$ is a projective generator in $\mathcal{M}_{A}$ and in ${ }_{A} \mathcal{M}$.

Theorem 0.2. Let $A \subset B \subset H$ where $H$ is a residually finite dimensional Hopf algebra, $B$ is a Hopf subalgebra, and $A$ is a right coideal subalgebra having semilocal localizations with respect to a central subring $Z$. Suppose $B$ is right module-finite over $A$ and the antipode of $B$ is bijective. Then $H$ is a projective generator in $\mathcal{M}_{A}$.

In both theorems we encounter projective modules of a very special kind. In fact $H \otimes_{A} A_{\mathfrak{m}}$ is a free $A_{\mathfrak{m}}$-module for any maximal ideal $\mathfrak{m}$ of $Z$. When $\operatorname{dim} H<\infty$, Theorem 0.2 applies to an arbitrary right coideal subalgebra $A$ since we may take $Z=k$. In this case $H$ is a free $A$-module, which generalizes the Nichols-Zoeller 
freeness theorem [22]. The investigation of the freeness over right coideal subalgebras in the finite dimensional case was initiated in [13], [17], and the full solution was obtained in [28].

Over coideal subalgebras one can expect a Hopf algebra to be a flat module rather than faithfully flat or projective. If $H=k[G]$ is the function algebra of an affine group scheme $G$ of finite type over $k$ then for any group subscheme $K$ of $G$ the function algebra $A=k[K \backslash G]$ on the right homogeneous space $K \backslash G$ is a right coideal subalgebra of $H$. There are many cases where $K \backslash G$ is quasiaffine, and so $K \backslash G$ may be identified with an open subscheme $U$ of the affine scheme $\operatorname{Spec} A$. Since the canonical morphism $G \rightarrow K \backslash G$ is flat, $H$ is always flat over $A$. However, the faithful flatness and projectivity are obtained precisely when $U=\operatorname{Spec} A$, i.e., when $K \backslash G$ is affine.

Theorem 0.3. Let $A \subset B \subset H$ where $H$ is a directed union of residually finite dimensional Hopf subalgebras, $B$ is any Hopf subalgebra, and $A$ is a right coideal subalgebra contained in the center of $B$. Then $H$ is flat in $\mathcal{M}_{A}$. If $A$ is a Hopf subalgebra then $H$ is a projective generator in $\mathcal{M}_{A}$ and in ${ }_{A} \mathcal{M}$.

Any commutative Hopf algebra $H$ is a directed union of finitely generated Hopf subalgebras, and those are residually finite dimensional. In this case Theorem 0.3 applies to an arbitrary right coideal subalgebra $A$; we recover the projectivity result of Takeuchi [32] and the flatness result of Masuoka and Wigner [18]. Our result is new even when $A$ is a central Hopf subalgebra of $H$. An interesting known example is the quantized function algebra at a root of unity; this Hopf algebra contains the ordinary function algebra of a semisimple algebraic group in its center. Projectivity was proved in this case by De Concini and Lyubashenko [10]; they needed detailed information about quantized function algebras.

Several related results are known where $H$ is not assumed to be residually finite dimensional, but there are restrictions of a different kind. As was established by Schneider [27], any left or right noetherian Hopf algebra is a faithfully flat module over central Hopf subalgebras. More recently Wu and Zhang [33] discovered that the projectivity holds for finite extensions of finitely generated PI Hopf algebras under certain finiteness assumptions about injective or projective dimensions. Of a somewhat different flavor are results for pointed Hopf algebras [14], [24] or Hopf algebras with cocommutative coradical [16] which impose a restriction on the coalgebra structure rather than the algebra structure.

As was emphasized in [28], it is natural to investigate projectivity in the more general settings where $A$ is assumed to be a (right) $H$-comodule algebra. Such an algebra $A$ has a right $H$-comodule structure given by an algebra homomorphism $\rho_{A}: A \rightarrow A \otimes H$. With $A$ one associates the category of right Hopf modules $\mathcal{M}_{A}^{H}[11]$, [32]. The objects of $\mathcal{M}_{A}^{H}$ have structures of a right $A$-module and a right $H$-comodule such that the comodule structure map $\rho_{M}: M \rightarrow M \otimes H$ becomes an $\mathcal{M}_{A}$-morphism if we let $A$ operate on $M \otimes H$ via $\rho_{A}$. When $A$ and $H$ are commutative, $\mathcal{M}_{A}^{H}$ is equivalent to the category of $G$-linearized quasicoherent sheaves on $\operatorname{Spec} A$, where $G$ is the group scheme corresponding to $H$. As usual we use the term " $H$-costable" in the sense "stable under the coaction of $H$ ".

Problem 1. Let $A$ be an $H$-simple $H$-comodule algebra, i.e., $A$ has no $H$-costable ideals other than 0 and $A$. For what classes of algebras is every nonzero object of $\mathcal{M}_{A}^{H}$ a projective generator in $\mathcal{M}_{A}$ ? 
When $H=k$ is the trivial Hopf algebra, the $H$-simplicity of $A$ means that $A$ is simple, and the question asks whether all right $A$-modules are projective. Certainly, this holds if and only if $A$ is artinian. In general Problem 1 is meaningful under the assumption that $A$ has a simple artinian factor ring. This is automatic for subalgebras of $H$ since the counit $\varepsilon: H \rightarrow k$ makes $k$ into a factor algebra of any subalgebra. In order to treat flatness we want to weaken the assumption about the $H$-simplicity of $A$.

Problem 2. Let $A$ be an $H$-costable subalgebra of an $H$-comodule algebra B. Suppose that $I B=B$ for each nonzero $H$-costable ideal $I$ of $A$. For what classes of algebras is every object of $\mathcal{M}_{B}^{H}$ flat in $\mathcal{M}_{A}$ ?

There is a dual formulation for (left) $H$-module algebras. Here $A$ is an algebra which has a left $H$-module structure compatible with the multiplication; $\mathcal{M}_{A}^{H}$ is replaced with the category ${ }_{H} \mathcal{M}_{A}$ whose objects have a right $A$-module structure and a compatible left $H$-module structure. Working with module algebras gives some advantage since in this case the coalgebra structure on $H$ is important, and we can use the family of finite dimensional subcoalgebras. In fact we are only able to approach Problems 1 and 2 for $H$-comodule algebras by making a reduction to similar questions for module algebras over the finite dual $H^{\circ}$ of $H$. The correct correspondence between the $H$-comodule structure and the $H^{\circ}$-module structure is available when $H$ is residually finite dimensional. This explains why this kind of restriction on $H$ appears in Theorems 0.1-0.3.

An object $M \in{ }_{H} \mathcal{M}_{A}$ is called $A$-finite if $M$ is finitely generated as an $A$-module; $M$ is locally A-finite if $M$ is a directed union of $A$-finite subobjects. Theorem 5.6 provides a projectivity result for locally $A$-finite objects of ${ }_{H} \mathcal{M}_{A}$ assuming that $A$ has semilocal localizations with respect to a central subring. This unifies the cases of commutative algebras and semilocal ones considered in [28].

The short proof of the previous result proposed in [28] for the case of commutative $A$ and cocommutative $H$ is based on three properties of the Fitting ideals of a finitely generated $A$-module $M$ : (1) these ideals contain enough information to recognize projective modules of constant rank, (2) they behave functorially with respect to the change of ring, (3) they are stable under a compatible action of $H$. The definition of the Fitting ideals involves computing determinants, so it does not generalize to noncommutative rings.

In section 1 of the present article we introduce certain ideals $I_{r}(M)$ of a ring $R$ for any rational number $r \geq 0$ and a finitely generated right $R$-module $M$ imposing some assumptions about the localizations $R_{\mathfrak{m}}$ at the maximal ideals of a central subring of $R$. There are analogs, though less satisfactory, of the three properties mentioned above. When $R=A$ is an $H$-module algebra with semilocal central localizations and $M \in{ }_{H} \mathcal{M}_{A}$, the ideals $I_{r}(M)$ are not $H$-stable in general. Our expectation is that the normalized rank $r_{P}(M)$ at a maximal ideal $P$ of $A$ is determined by those rational values $r$ for which $P$ contains the smallest $H$-stable ideal $J_{r}(M)$ of $A$ such that $I_{r}(M) \subset J_{r}(M)$. When this holds, $r_{P}(M)=r_{Q}(M)$ for any pair $P, Q$ of maximal ideals of $A$ containing the same $H$-stable ideals. This property can be viewed as the $H$-invariance of the rank function $P \mapsto r_{P}(M)$ defined on the maximal spectrum Max $A$ of $A$. We are able to prove it only under some technical restrictions. As a result, we gain less control over the situation in those cases where $A$ is not $H$-simple. This leads to more restrictive assumptions about $A$ when dealing with flatness. 
We will use standard notation from the theory of Hopf algebras [19], [30]. For each ring $R$ denote by $\operatorname{Jac}(R)$ the Jacobson radical of $R$, by Max $R$ and Spec $R$ the maximal and prime spectra of $R$. A ring $R$ is semilocal if $R / \operatorname{Jac}(R)$ is artinian; a semilocal ring with a single maximal ideal is quasilocal. A semilocal ring $R$ is semiprimary if $\operatorname{Jac}(R)$ is nilpotent; a semiprimary ring with a single maximal ideal is primary. Denote by $\mathbb{Z}_{+}$the semigroup of positive integers.

\section{Construction of ideals}

Let $R$ be a ring. If $M \in \mathcal{M}_{R}$ is generated by elements $e_{1}, \ldots, e_{n}$, we denote by $\mathcal{I}_{e_{1}, \ldots, e_{n}}$ the ideal of $R$ generated by all elements of $R$ which occur as a coefficient in a zero linear combination $e_{1} x_{1}+\cdots+e_{n} x_{n}=0$ with $x_{1}, \ldots, x_{n} \in R$.

Lemma 1.1. Suppose that $e_{1}, \ldots, e_{n}$ generate $M \in \mathcal{M}_{R}$. If $\varphi: R \rightarrow R^{\prime}$ is a ring homomorphism and $e_{i}^{\prime}=e_{i} \otimes 1 \in M \otimes_{R} R^{\prime}$, then $\mathcal{I}_{e_{1}^{\prime}, \ldots, e_{n}^{\prime}}=R^{\prime} \varphi\left(\mathcal{I}_{e_{1}, \ldots, e_{n}}\right) R^{\prime}$.

Proof. Let $\theta: R^{n} \rightarrow M$ be the epimorphism in $\mathcal{M}_{R}$ sending the standard generators of the free module $R^{n}$ to $e_{1}, \ldots, e_{n}$. For $i=1, \ldots, n$ denote by $\pi_{i}: R^{n} \rightarrow R$ the projection onto the $i$ th summand. The ideal $\mathcal{I}_{e_{1}, \ldots, e_{n}}$ is generated by $\sum \pi_{i}(K)$ where $K=\operatorname{Ker} \theta$. Tensoring with $R^{\prime}$, we get an exact sequence of $R^{\prime}$-modules

$$
K \otimes_{R} R^{\prime} \stackrel{\alpha \otimes \mathrm{id}}{\longrightarrow} R^{n} \otimes_{R} R^{\prime} \stackrel{\theta \otimes \mathrm{id}}{\longrightarrow} M \otimes_{R} R^{\prime} \rightarrow 0
$$

where $\alpha: K \rightarrow R^{n}$ is the inclusion map. We have an isomorphism $R^{n} \otimes_{R} R^{\prime} \cong R^{\prime n}$ with $\pi_{i} \otimes$ id $: R^{n} \otimes_{R} R^{\prime} \rightarrow R^{\prime}$ giving the projection onto the $i$ th summand. Hence $\mathcal{I}_{e_{1}^{\prime}, \ldots, e_{n}^{\prime}}$ coincides with the ideal of $R^{\prime}$ generated by $\sum\left(\pi_{i} \otimes \mathrm{id}\right)\left(K^{\prime}\right)$ where $K^{\prime}=$ $\operatorname{Ker}(\theta \otimes \mathrm{id})$. Since $K^{\prime}$ is equal to the image of $\alpha \otimes \mathrm{id}$, the conclusion is clear.

Recall that $R$ is said to be weakly finite if for each integer $n>0$ every generating set for the free right $R$-module $R^{n}$ containing exactly $n$ elements is a basis for $R^{n}$; equivalently, every $R$-module epimorphism $R^{n} \rightarrow R^{n}$ is an isomorphism. This can also be reformulated in terms of invertibility of $n \times n$-matrices with entries in $R$.

Lemma 1.2. Suppose that all factor rings of $R$ are weakly finite. If $e_{1}, \ldots, e_{n}$ and $e_{1}^{\prime}, \ldots, e_{n}^{\prime}$ are two systems of generators for $M$ having the same number of elements then $\mathcal{I}_{e_{1}^{\prime}, \ldots, e_{n}^{\prime}}=\mathcal{I}_{e_{1}, \ldots, e_{n}}$.

Proof. This follows from [28, Lemma 2.3]. By that lemma $\mathcal{I}_{e_{1}^{\prime}, \ldots, e_{n}^{\prime}} \subset \mathcal{I}_{e_{1}, \ldots, e_{n}}$ since $R / \mathcal{I}_{e_{1}, \ldots, e_{n}}$ is weakly $n$-finite. The opposite inclusion holds by symmetry.

If $R$ has weakly finite factor rings and $M$ is $n$-generated then we put

$$
I_{n}(M)=\mathcal{I}_{e_{1}, \ldots, e_{n}}
$$

where $e_{1}, \ldots, e_{n}$ is any set of $n$ generators for $M$. By Lemma 1.2 the above ideal does not depend on the choice of a generating set. The ideals $I_{r}(M)$ are thus defined for all integers $r \geq \mu(M)$ where $\mu(M)$ denotes the minimal number of generators for $M$. When $M=0$ we put $\mu(M)=0$ and $I_{0}(M)=0$ for consistency reasons.

We do not indicate the base ring explicitly in the notation for $I_{r}(M)$. Given a ring homomorphism $R \rightarrow R^{\prime}$, let $I_{r}\left(M \otimes_{R} R^{\prime}\right)$ be the ideal of $R^{\prime}$ corresponding to the induced $R^{\prime}$-module $M \otimes_{R} R^{\prime}$ (when defined). Especially, this convention will be in force when $R^{\prime}$ is either a factor ring or an Ore localization of $R$. 
Lemma 1.3. Suppose that $R, R^{\prime}$ are two rings with weakly finite factor rings, $\varphi: R \rightarrow R^{\prime}$ a homomorphism and $M, N$ two finitely generated right $R$-modules.

(i) $I_{r}(M)=R$ for all integers $r>\mu(M)$.

(ii) $M \cong R^{n}$ if and only if $M$ is n-generated with $I_{n}(M)=0$.

(iii) $I_{r+s}(M \oplus N)=I_{r}(M)+I_{s}(N)$ for all integers $r \geq \mu(M)$ and $s \geq \mu(N)$.

(iv) $I_{r t}\left(M^{t}\right)=I_{r}(M)$ for all integers $r \geq \mu(M)$ and $t>0$.

(v) $I_{r}\left(M \otimes_{R} R^{\prime}\right)=R^{\prime} \varphi\left(I_{r}(M)\right) R^{\prime}$ for all integers $r \geq \mu(M)$.

Proof. (i) If $r>\mu(M)$ then $M$ can be generated by $r-1$ elements, say $e_{1}, \ldots, e_{r-1}$. Adding another element $e_{r}=0$, we get a set of $r$ generators for $M$. Now $I_{r}(M)=$ $\mathcal{I}_{e_{1}, \ldots, e_{r}}$. However, $1 \in \mathcal{I}_{e_{1}, \ldots, e_{r}}$ since 1 is a coefficient in the relation $e_{r}=0$.

(ii) Let $M$ be generated by $e_{1}, \ldots, e_{n}$. Clearly, $e_{1}, \ldots, e_{n}$ is a basis for $M$ if and only if $\mathcal{I}_{e_{1}, \ldots, e_{n}}=0$.

(iii) Let us identify $M$ and $N$ with submodules of $M \oplus N$. Pick generating sets $e_{1}, \ldots, e_{r}$ for $M$ and $e_{1}^{\prime}, \ldots, e_{s}^{\prime}$ for $N$. Then the $r+s$ elements $e_{1}, \ldots, e_{r}, e_{1}^{\prime}, \ldots, e_{s}^{\prime}$ generate $M \oplus N$. Given $x_{1}, \ldots, x_{r}, y_{1}, \ldots, y_{s} \in R$, the equality $\sum e_{i} x_{i}+\sum e_{j}^{\prime} y_{j}=0$ holds if and only if both $\sum e_{i} x_{i}=0$ and $\sum e_{j}^{\prime} y_{j}=0$. Hence

$$
\mathcal{I}_{e_{1}, \ldots, e_{r}, e_{1}^{\prime}, \ldots, e_{s}^{\prime}}=\mathcal{I}_{e_{1}, \ldots, e_{r}}+\mathcal{I}_{e_{1}^{\prime}, \ldots, e_{s}^{\prime}}
$$

(iv) This follows from (iii) by induction on $t$.

(v) This is a restatement of Lemma 1.1.

Suppose that $M \in \mathcal{M}_{R}$ is finitely generated and $r \geq 0$ is a rational number. The set $\left\{l \in \mathbb{Z}_{+} \mid r l \geq \mu\left(M^{l}\right)\right\}$ is closed under addition since $\mu\left(M^{l+t}\right) \leq \mu\left(M^{l}\right)+\mu\left(M^{t}\right)$ for all $l, t \in \mathbb{Z}_{+}$. If the inequality $r l \geq \mu\left(M^{l}\right)$ holds for at least one $l$ then we can find such an $l$ with the property that $r l \in \mathbb{Z}$, replacing $l$ with a suitable multiple if necessary. We put then

$$
I_{r}(M)=I_{r l}\left(M^{l}\right),
$$

which does not depend on the choice of an $l$ with the above properties. In fact, if $t \in \mathbb{Z}_{+}$also satisfies $r t \in \mathbb{Z}$ and $r t \geq \mu\left(M^{t}\right)$ then $I_{r l}\left(M^{l}\right)=I_{r l t}\left(M^{l t}\right)=I_{r t}\left(M^{t}\right)$ by (iv) of Lemma 1.3. If $r \in \mathbb{Z}$ and $r \geq \mu(M)$ then the initial definition of $I_{r}(M)$ agrees with the newer one since $l=1$ satisfies the required properties.

We say that $r$ is $M$-admissible if $r l \geq \mu\left(M^{l}\right)$ for some $l \in \mathbb{Z}_{+}$. We have defined the ideals $I_{r}(M)$ for all $M$-admissible rational numbers. All statements in the next lemma immediately reduce to the corresponding statements in Lemma 1.3.

Lemma 1.4. Retaining the assumptions about $R, R^{\prime}, \varphi, M, N$ as in Lemma 1.3, let $r \in \mathbb{Q}$ be $M$-admissible and $s \in \mathbb{Q}$ be $N$-admissible.

(i) $I_{r}(M)=R$ whenever $r l>\mu\left(M^{l}\right)$ for some $l \in \mathbb{Z}_{+}$.

(ii) $M^{l} \cong R^{n}$ for integers $l>0, n \geq 0$ if and only if $n \geq \mu\left(M^{l}\right)$ and $I_{n / l}(M)=0$.

(iii) $I_{r+s}(M \oplus N)=I_{r}(M)+I_{s}(N)$.

(iv) $I_{r t}\left(M^{t}\right)=I_{r}(M)$ for all $t \in \mathbb{Z}_{+}$.

(v) $I_{r}\left(M \otimes_{R} R^{\prime}\right)=R^{\prime} \varphi\left(I_{r}(M)\right) R^{\prime}$.

Part (v) of this lemma is valid in a slightly more general situation where $R$ is not assumed to have weakly finite factor rings. 
Lemma 1.5. Suppose that $r \in \mathbb{Q}$ is $M$-admissible. Then $R$ has an ideal $K$ such that $I_{r}\left(M \otimes_{R} R^{\prime}\right)=R^{\prime} \varphi(K) R^{\prime}$ for any homomorphism $\varphi: R \rightarrow R^{\prime}$ into a ring $R^{\prime}$ with weakly finite factor rings.

Proof. There exists $l \in \mathbb{Z}_{+}$such that $n=r l \in \mathbb{Z}$ and $n \geq \mu\left(M^{l}\right)$. Then $M^{l}$ is generated by $n$ elements, say $e_{1}, \ldots, e_{n}$. Take $K=\mathcal{I}_{e_{1}, \ldots, e_{n}}$. For any $\varphi$ satisfying the hypotheses the $R^{\prime}$-module $M^{l} \otimes_{R} R^{\prime}$ is generated by $e_{1}^{\prime}, \ldots, e_{n}^{\prime}$ where $e_{i}^{\prime}=e_{i} \otimes 1$ for each $i$. Lemma 1.1 yields $I_{r}\left(M \otimes_{R} R^{\prime}\right)=I_{n}\left(M^{l} \otimes_{R} R^{\prime}\right)=\mathcal{I}_{e_{1}^{\prime}, \ldots, e_{n}^{\prime}}=R^{\prime} \varphi(K) R^{\prime}$.

If $M \in \mathcal{M}_{R}$ is finitely generated and $P \in \operatorname{Max} R$ is such that $R / P$ is simple artinian, then we put

$$
r_{P}(M)=\frac{\text { length } M / M P}{\text { length } R / P}
$$

where length stands for the composition series length in $\mathcal{M}_{R}$.

If $R$ is semilocal then the set $\operatorname{Max} R$ is finite and coincides with the set of primitive ideals of $R$. For each $P \in \operatorname{Max} R$ the ring $R / P$ is simple artinian, so that $r_{P}(M)$ is defined. Recall that any semilocal ring is weakly finite. Moreover, all factor rings of such a ring are themselves semilocal, hence weakly finite.

Lemma 1.6. Suppose $R$ is semilocal, $M \in \mathcal{M}_{R}$ is finitely generated and $r \in \mathbb{Q}$.

(i) $\mu(M)=\min \left\{n \in \mathbb{Z} \mid n \geq r_{P}(M)\right.$ for all $\left.P \in \operatorname{Max} R\right\}$.

(ii) $r$ is $M$-admissible if and only if $r \geq r_{P}(M)$ for all $P \in \operatorname{Max} R$.

Proof. (i) Let $J=\operatorname{Jac}(R)$. We have $M / M J \cong \prod_{P \in \operatorname{Max} R} M / M P$ since $R / J$ is semisimple artinian. It follows from Nakayama's Lemma that $M$ is $n$-generated if and only if so is $M / M P$ for each $P$. Since every $R / P$-module is isomorphic to a direct sum of copies of the simple module, $M / M P$ is an epimorphic image of $(R / P)^{n}$ if and only if length $M / M P \leq$ length $(R / P)^{n}$, which can be rewritten as $r_{P}(M) \leq n$.

(ii) Put $m=\max \left\{r_{P}(M) \mid P \in \operatorname{Max} R\right\}$. It was proved in (i) that $\mu\left(M^{l}\right)$ is equal to the smallest integer $n$ such that $n \geq r_{P}\left(M^{l}\right)=r_{P}(M) l$ for all $P$, i.e., $n \geq m l$. Thus $r l \geq \mu\left(M^{l}\right)$ implies $r \geq m$. If $r=m$ then the required inequality holds for any $l \in \mathbb{Z}_{+}$such that $m l \in \mathbb{Z}$.

Let $Z \subset R$ be a central subring. Denote by $R_{z}$ and $R_{\mathfrak{p}}$, respectively, the localizations of $R$ at the multiplicatively closed subsets $\left\{z^{i} \mid i=0,1, \ldots\right\}$ and $Z \backslash \mathfrak{p}$ where $z \in Z$ is any element and $\mathfrak{p}$ a prime ideal of $Z$. Similarly, $M_{z}$ and $M_{\mathfrak{p}}$ will denote the respective localizations of $M \in \mathcal{M}_{R}$. If $M$ is finitely generated, then $M_{z}$ is a finitely generated $R_{z}$-module and $M_{\mathfrak{p}}$ a finitely generated $R_{\mathfrak{p}}$-module. For each rational number $r \geq 0$ put

$$
\begin{gathered}
T_{r}(M)=\left\{z \in Z \mid r \text { is } M_{z} \text {-admissible }\right\}, \\
\widetilde{U}_{r}(M)=\left\{\mathfrak{p} \in \operatorname{Spec} Z \mid r \text { is } M_{\mathfrak{p}} \text {-admissible }\right\}, \\
U_{r}(M)=\left\{\mathfrak{m} \in \operatorname{Max} Z \mid r \text { is } M_{\mathfrak{m}} \text {-admissible }\right\} .
\end{gathered}
$$

The open subsets $D(z)=\{\mathfrak{p} \in \operatorname{Spec} Z \mid z \notin \mathfrak{p}\}$ with $z \in Z$ give a basis for the topology on Spec $Z$. If $\mathfrak{p} \in D(z)$ then $R_{\mathfrak{p}}$ is a localization of $R_{z}$ and $M_{\mathfrak{p}} \cong M_{z} \otimes_{R_{z}} R_{\mathfrak{p}}$, whence $\mu\left(M_{\mathfrak{p}}^{l}\right) \leq \mu\left(M_{z}^{l}\right)$ for each $l \in \mathbb{Z}_{+}$. It follows that $D(z) \subset \widetilde{U}_{r}(M)$ whenever $z \in T_{r}(M)$. 
Lemma 1.7. Any $\mathfrak{p} \in \widetilde{U}_{r}(M)$ is contained in $D(z)$ for some $z \in T_{r}(M)$. Hence $\widetilde{U}_{r}(M)$ is open in $\operatorname{Spec} Z$ and $U_{r}(M)=\widetilde{U}_{r}(M) \cap \operatorname{Max} Z$ is open in $\operatorname{Max} Z$.

Proof. Let $l>0$ be an integer such that $n=r l \in \mathbb{Z}$ and $n \geq \mu\left(M_{\mathfrak{p}}^{l}\right)$. We can find $n$ elements $e_{1}, \ldots, e_{n} \in M^{l}$ whose images in $M_{\mathfrak{p}}^{l}$ generate the latter $R_{\mathfrak{p}}$-module. For each $x \in M^{l}$ there exists $z \in Z \backslash \mathfrak{p}$ such that $x z$ is an $R$-linear combination of $e_{1}, \ldots, e_{n}$. As $M^{l}$ is finitely generated, we can find a $z$ which fulfills the required property for all $x$ simultaneously. Then the $R_{z}$-module $M_{z}^{l}$ is generated by the images of $e_{1}, \ldots, e_{n}$, whence $n \geq \mu\left(M_{z}^{l}\right)$. Thus $z \in T_{r}(M)$ and $D(z)$ is a neighborhood of $\mathfrak{p}$ contained in $\widetilde{U}_{r}(M)$.

Lemma 1.8. Suppose that $\mathfrak{m} R_{\mathfrak{m}} \subset \operatorname{Jac}\left(R_{\mathfrak{m}}\right)$ for each $\mathfrak{m} \in \operatorname{Max} Z$. Then any finite subset $X \subset U_{r}(M)$ is contained in $D(z)$ for some $z \in T_{r}(M)$.

Proof. We can find $l \in \mathbb{Z}_{+}$such that $n=r l \in \mathbb{Z}$ and $n \geq \mu\left(M_{\mathfrak{m}}^{l}\right)$ for all $\mathfrak{m} \in X$. Since $M_{\mathfrak{m}} / M_{\mathfrak{m}} \mathfrak{m} \cong M / M \mathfrak{m}$, we have $n \geq \mu\left(M^{l} / M^{l} \mathfrak{m}\right)$ for $\mathfrak{m} \in X$. Since $\mathfrak{m}+\mathfrak{n}=Z$ for any pair of distinct ideals $\mathfrak{m}, \mathfrak{n} \in X$, the canonical map $M^{l} \rightarrow \prod_{\mathfrak{m} \in X} M^{l} / M^{l} \mathfrak{m}$ is surjective by Chinese Remainder Theorem. There exist $e_{1}, \ldots, e_{n} \in M^{l}$ whose cosets modulo $\mathfrak{m}$ generate the $R$-module $M^{l} / M^{l} \mathfrak{m}$ for each $\mathfrak{m} \in X$. Then for each $\mathfrak{m} \in X$ the images of $e_{1}, \ldots, e_{n}$ in $M_{\mathfrak{m}}^{l}$ generate the $R_{\mathfrak{m}}$-module $M_{\mathfrak{m}}^{l}$ modulo $M_{\mathfrak{m}}^{l} \mathfrak{m}$; by Nakayama's Lemma $M_{\mathfrak{m}}^{l}$ is generated by those images.

For $x \in M^{l}$ denote by $\mathfrak{a}_{x}$ the ideal of $Z$ consisting of those elements $z \in Z$ for which $x z$ lies in the submodule $N$ of $M^{l}$ generated by $e_{1}, \ldots, e_{n}$. Since $N_{\mathfrak{m}}=M_{\mathfrak{m}}^{l}$, we have $\mathfrak{a}_{x} \not \subset \mathfrak{m}$ for any $\mathfrak{m} \in X$; hence $\mathfrak{a}_{x} \not \subset \bigcup_{\mathfrak{m} \in X} \mathfrak{m}$ [4, Ch. II, §1, Prop. 2]. So there exists $z \in Z$ which lies in none of the ideals from $X$ and satisfies $x z \in N$. As $M^{l}$ is finitely generated, we can find a $z$ which fulfills that property for all $x$ simultaneously. We obtain $z \in T_{r}(M)$ and $X \subset D(z)$.

Further on in this section we make the following assumption:

(A) $R_{\mathfrak{m}}$ has weakly finite factor rings and $\mathfrak{m} R_{\mathfrak{m}} \subset \operatorname{Jac}\left(R_{\mathfrak{m}}\right)$ for each $\mathfrak{m} \in \operatorname{Max} Z$.

Since $R$ is embedded in $\prod_{\mathfrak{m} \in \operatorname{Max} Z} R_{\mathfrak{m}}$, the weak finiteness of $R$ follows from the weak finiteness of all localizations $R_{\mathfrak{m}}$. This observation, applied to the factor rings of $R$, shows that $R$ has weakly finite factor rings provided that so do all rings $R_{\mathfrak{m}}$.

Let $M \in \mathcal{M}_{R}$ be finitely generated and $r \in \mathbb{Q}$. When $r$ is $M$-admissible, we have $U_{r}(M)=\operatorname{Max} Z$ and $I_{r}\left(M_{\mathfrak{m}}\right)=I_{r}(M) R_{\mathfrak{m}}$ for all $\mathfrak{m} \in \operatorname{Max} Z$ by Lemma 1.4(v). Every ideal of $R$ is completely determined by its extensions to the rings $R_{\mathfrak{m}}$. In particular, $I_{r}(M)$ consists precisely of those elements $a \in R$ whose image $a_{\mathfrak{m}}$ in $R_{\mathfrak{m}}$ belongs to $I_{r}\left(M_{\mathfrak{m}}\right)$ for each $\mathfrak{m} \in \operatorname{Max} Z$.

We now extend the range of $r$ in the definition of $I_{r}(M)$ to arbitrary nonnegative values. The ideal $I_{r}\left(M_{\mathfrak{m}}\right)$ of $R_{\mathfrak{m}}$ has already been defined when $\mathfrak{m} \in U_{r}(M)$. Put

$$
I_{r}(M)=\left\{a \in R \mid a_{\mathfrak{m}} \in I_{r}\left(M_{\mathfrak{m}}\right) \text { for each } \mathfrak{m} \in U_{r}(M)\right\} .
$$

Clearly $I_{r}(M)$ is an ideal of $R$. If $U_{r}(M)=\varnothing$ then $I_{r}(M)=R$.

Lemma 1.9. If $U_{r}(M)$ is quasicompact, then $I_{r}(M) R_{\mathfrak{m}}=I_{r}\left(M_{\mathfrak{m}}\right)$ for $\mathfrak{m} \in U_{r}(M)$.

Proof. The ideals of $R_{\mathfrak{m}}$ are extensions of ideals of $R$. Fixing $\mathfrak{m}$, we have to show that for each $a \in R$ with $a_{\mathfrak{m}} \in I_{r}\left(M_{\mathfrak{m}}\right)$ there exists $s \in Z \backslash \mathfrak{m}$ such that $a s \in I_{r}(M)$, i.e., $a_{\mathfrak{n}} s_{\mathfrak{n}} \in I_{r}\left(M_{\mathfrak{n}}\right)$ for all $\mathfrak{n} \in U_{r}(M)$. 
Suppose that $\mathfrak{m} \in D(z)$ where $z \in T_{r}(M)$. As $r$ is $M_{z}$-admissible, Lemma 1.5 can be applied to the $R_{z}$-module $M_{z}$. Let $K$ be the ideal of $R_{z}$ given by that lemma. For each $\mathfrak{n} \in D(z) \cap \operatorname{Max} Z$ the ring $R_{\mathfrak{n}}$ is a localization of $R_{z}$, whence $I_{r}\left(M_{\mathfrak{n}}\right)=K R_{\mathfrak{n}}$ by Lemma 1.5. Applying this formula with $\mathfrak{m}=\mathfrak{n}$, we deduce that $a_{z} t_{z} \in K$ for a suitable $t \in Z \backslash \mathfrak{m}$ where $a_{z}, t_{z}$ denote the images of $a, t$ in $R_{z}$. Passing now to $R_{\mathfrak{n}}$, we see that $a_{\mathfrak{n}} t_{\mathfrak{n}} \in I_{r}\left(M_{\mathfrak{n}}\right)$ for any $\mathfrak{n}$ as above.

Given an arbitrary $\mathfrak{n} \in U_{r}(M)$, there exists $z \in T_{r}(M)$ such that $\{\mathfrak{m}, \mathfrak{n}\} \subset D(z)$ by Lemma 1.8. Hence $U_{r}(M)$ is covered by the open subsets $D(z)$ with $z \in T_{r}(M)$, $z \notin \mathfrak{m}$. Since $U_{r}(M)$ is quasicompact, we have $U_{r}(M) \subset D\left(z_{1}\right) \cup \cdots \cup D\left(z_{n}\right)$ for some elements $z_{1}, \ldots, z_{n} \in T_{r}(M)$ such that $\mathfrak{m} \in D\left(z_{i}\right)$ for each $i=1, \ldots, n$. We have seen that for each $i$ there exists $t_{i} \in Z \backslash \mathfrak{m}$ such that $a_{\mathfrak{n}}\left(t_{i}\right)_{\mathfrak{n}} \in I_{r}\left(M_{\mathfrak{n}}\right)$ for all $\mathfrak{n} \in D\left(z_{i}\right) \cap \operatorname{Max} Z$. Now $s=t_{1} \cdots t_{n}$ is the desired element.

Remark. The topological space $\operatorname{Max} Z$ is always quasicompact. Hence Lemma 1.9 applies for any $r$ with $U_{r}(M)=\operatorname{Max} Z$. If $\operatorname{Max} Z$ is noetherian (e.g., if $Z / \operatorname{Jac}(Z)$ is noetherian), then every open subset of $\operatorname{Max} Z$ is quasicompact. In this case any nonnegative value of $r$ is legitimate.

Lemma 1.10. Let $\varphi: R \rightarrow R^{\prime}$ be a ring homomorphism where $R$ satisfies (A), while $R^{\prime}$ has weakly finite factor rings. Suppose that $U_{r}(M)$ is quasicompact and there exists a finite subset $X \subset U_{r}(M)$ such that $\varphi(z)$ is invertible in $R^{\prime}$ for each $z \in Z \backslash \bigcup_{\mathfrak{m} \in X} \mathfrak{m}$. Then $I_{r}\left(M \otimes_{R} R^{\prime}\right)=R^{\prime} \varphi\left(I_{r}(M)\right) R^{\prime}$.

Proof. By Lemma 1.8 there exists $z \in T_{r}(M)$ such that $X \subset D(z)$, so that $z$ lies in none of the ideals $\mathfrak{m} \in X$. Since $\varphi(z)$ is invertible, $\varphi$ extends to a homomorphism $\psi: R_{z} \rightarrow R^{\prime}$. Recall that $r$ is $M_{z}$-admissible by the definition of $T_{r}(M)$. Let $K$ be the ideal of $R_{z}$ given by Lemma 1.5, when applied to the $R_{z}$-module $M_{z}$. Then $I_{r}\left(M \otimes_{R} R^{\prime}\right)=R^{\prime} \psi(K) R^{\prime}$, and also $I_{r}\left(M_{\mathfrak{m}}\right)=K R_{\mathfrak{m}}$ for each $\mathfrak{m} \in X$ as in the proof of Lemma 1.9. Thus the two ideals $K$ and $I_{r}(M) R_{z}$ of $R_{z}$ have the same extension to each ring $R_{\mathfrak{m}}$ with $\mathfrak{m} \in X$. Given any $a \in K$, there exists therefore $s \in Z \backslash \bigcup_{\mathfrak{m} \in X} \mathfrak{m}$ such that $a s_{z} \in I_{r}(M) R_{z}$ where $s_{z}$ denotes the image of $s$ in $R_{z}$. Since $\psi\left(s_{z}\right)=\varphi(s)$ is invertible in $R^{\prime}$, we deduce that $\psi(a) \in \psi\left(I_{r}(M) R_{z}\right) R^{\prime}=$ $\varphi\left(I_{r}(M)\right) R^{\prime}$. Similarly, given any $a \in I_{r}(M)$, we prove that $\varphi(a) \in \psi(K) R^{\prime}$. Hence $R^{\prime} \psi(K) R^{\prime}=R^{\prime} \varphi\left(I_{r}(M)\right) R^{\prime}$, and we are done.

Remark. Lemma 1.10 will be used in the special case where $\varphi$ is the canonical homomorphism onto a factor ring $R^{\prime}$ of $R$. Suppose that $R^{\prime}$ has finitely many maximal ideals and $\varphi^{-1}(P) \cap Z \in U_{r}(M)$ for each $P \in \operatorname{Max} R^{\prime}$. Take

$$
X=\left\{\varphi^{-1}(P) \cap Z \mid P \in \operatorname{Max} R^{\prime}\right\} .
$$

If $z \in Z$, then $\varphi(z) R^{\prime}$ is an ideal of $R^{\prime}$; hence either $\varphi(z) R^{\prime}=R^{\prime}$ or $\varphi(z) R^{\prime}$ is contained in some $P \in \operatorname{Max} R^{\prime}$. In the former case $\varphi(z)$ is invertible, while in the latter case $z \in \mathfrak{m}$ for some $\mathfrak{m} \in X$. When $U_{r}(M)$ is quasicompact and $R^{\prime}$ has weakly finite factor rings, the hypotheses of Lemma 1.10 are satisfied.

Note that $U_{r}(M) \subset U_{s}(M)$ whenever $r, s \in \mathbb{Q}$ satisfy $0 \leq r \leq s$. Put

$$
\lambda(M)=\inf \left\{r \in \mathbb{Q} \mid U_{r}(M)=\operatorname{Max} Z\right\} .
$$

Lemma 1.11. Suppose $R$ satisfies (A), $M$ is finitely generated and $r \in \mathbb{Q}, r \geq 0$. 
(i) $I_{r}(M)=R$ whenever $r>\lambda(M)$.

(ii) If $U_{r}(M)$ is quasicompact and $I_{r}(M)=0$ then for each $\mathfrak{m} \in U_{r}(M)$ there exist integers $l>0$ and $n \geq 0$ such that $r=n / l$ and $M_{\mathfrak{m}}^{l} \cong R_{\mathfrak{m}}^{n}$ in $\mathcal{M}_{R_{\mathfrak{m}}}$.

(iii) $I_{r t}\left(M^{t}\right)=I_{r}(M)$ for all $t \in \mathbb{Z}_{+}$.

Proof. (i) Pick any $s \in \mathbb{Q}$ such that $\lambda(M)<s<r$. Then $U_{s}(M)=\operatorname{Max} Z$. For each $\mathfrak{m} \in \operatorname{Max} Z$ there exists $l \in \mathbb{Z}_{+}$such that $r l>s l \geq \mu\left(M_{\mathfrak{m}}^{l}\right)$; Lemma 1.4(i) shows that $I_{r}\left(M_{\mathfrak{m}}\right)=R_{\mathfrak{m}}$. The conclusion is now immediate from the definition of $I_{r}(M)$.

(ii) If $\mathfrak{m} \in U_{r}(M)$, then there exists $l \in \mathbb{Z}_{+}$such that $n=r l \in \mathbb{Z}$ and $n \geq \mu\left(M_{\mathfrak{m}}^{l}\right)$. By Lemma $1.9 I_{r}\left(M_{\mathfrak{m}}\right)=0$. Now we may apply Lemma 1.4(ii).

(iii) Note that $U_{r t}\left(M^{t}\right)=U_{r}(M)$ by a straightforward check and $I_{r t}\left(M_{\mathfrak{m}}^{t}\right)=$ $I_{r}\left(M_{\mathfrak{m}}\right)$ for each $\mathfrak{m} \in U_{r}(M)$ by Lemma 1.4(iv).

Remark. If the ring $R_{\mathfrak{m}}$ is semilocal, then the isomorphism $M_{\mathfrak{m}}^{l} \cong R_{\mathfrak{m}}^{n}$ in (ii) holds for any pair of integers $l>0$ and $n \geq 0$ such that $r=n / l$.

\section{Rings with semilocal central localizations}

We will assume throughout the whole section that $R$ has semilocal localizations with respect to a central subring $Z$. Thus

$$
R_{\mathfrak{m}} \text { is semilocal and } \mathfrak{m} R_{\mathfrak{m}} \subset \operatorname{Jac}\left(R_{\mathfrak{m}}\right) \text { for each } \mathfrak{m} \in \operatorname{Max} Z \text {. }
$$

Note that $R$ satisfies assumption (A) from section 1 . Any factor ring $R^{\prime}$ of $R$ has semilocal localizations with respect to the image of $Z$ in $R^{\prime}$. In this section several properties of the ring $R$ will be stated for future use. Some of those are more or less known.

Lemma 2.1. For any right primitive ideal $P$ of $R$ the ring $R / P$ is simple artinian and $P \cap Z \in \operatorname{Max} Z$. Given $\mathfrak{m} \in \operatorname{Max} Z$, there are finitely many elements in the set

$$
\operatorname{Max}_{\mathfrak{m}} R=\{P \in \operatorname{Max} R \mid P \cap Z=\mathfrak{m}\} .
$$

The maximal ideals of $R_{\mathfrak{m}}$ are precisely the ideals $P_{\mathfrak{m}}=R_{\mathfrak{m}} P$ with $P \in \operatorname{Max}_{\mathfrak{m}} R$.

Proof. Let $P$ be the annihilator of a simple right $R$-module $V$. Since $1 \notin P$, there exists $\mathfrak{m} \in \operatorname{Max} Z$ such that $P \cap Z \subset \mathfrak{m}$. The transformation $z_{V}$ of $V$ afforded by an element $z \in Z$ is an $\mathcal{M}_{R^{-}}$endomorphism. Hence the image and the kernel of $z_{V}$ are submodules of $V$. If $z \notin \mathfrak{m}$, then $V z \neq 0$, whence $V z=V$ and Ker $z_{V}=0$ by the simplicity of $V$. In other words, $z_{V}$ is invertible for any $z \in Z \backslash \mathfrak{m}$. We may now regard $V$ as a simple $R_{\mathfrak{m}}$-module. The condition $\mathfrak{m} R_{\mathfrak{m}} \subset \operatorname{Jac}\left(R_{\mathfrak{m}}\right)$ entails $V \mathfrak{m}=0$, i.e., $\mathfrak{m} \subset P$. The maximality of $\mathfrak{m}$ yields $P \cap Z=\mathfrak{m}$. Now $P / \mathfrak{m} R$ is a right primitive ideal of the factor $\operatorname{ring} R / \mathfrak{m} R \cong R_{\mathfrak{m}} / \mathfrak{m} R_{\mathfrak{m}}$. The latter is semilocal since so is $R_{\mathfrak{m}}$. Hence $R / \mathfrak{m} R$ has finitely many primitive ideals, and the factor algebra by any of those is simple artinian.

If $P$ is any ideal of $R$ such that $\mathfrak{m} \subset P$ for some $\mathfrak{m} \in \operatorname{Max} Z$, then $R_{\mathfrak{m}} / P_{\mathfrak{m}} \cong$ $(R / P)_{\mathfrak{m}} \cong R / P$; in this case $P_{\mathfrak{m}} \in \operatorname{Max} R_{\mathfrak{m}}$ if and only if $P \in \operatorname{Max}_{\mathfrak{m}} R$. Any ideal $P^{\prime}$ of $R_{\mathfrak{m}}$ coincides with $P_{\mathfrak{m}}$ where $P$ is the preimage of $P^{\prime}$ in $R$; if $P^{\prime} \in \operatorname{Max} R_{\mathfrak{m}}$, then $\operatorname{Jac}\left(R_{\mathfrak{m}}\right) \subset P^{\prime}$, and the assumption about $R_{\mathfrak{m}}$ yields $\mathfrak{m} \subset P$, so that $P \in \operatorname{Max}_{\mathfrak{m}} R$.

Suppose further that $M$ is a finitely generated right $R$-module. 
Lemma 2.2. If $M \mathfrak{a}=M$ for some ideal $\mathfrak{a}$ of $Z$ then there exists $a \in \mathfrak{a}$ such that $M(1-a)=0$. In particular, $\mathfrak{a}=Z$ whenever $M$ is faithful.

Proof. For each $\mathfrak{m} \in \operatorname{Max} Z$ the right $R_{\mathfrak{m}}$-module $M_{\mathfrak{m}}$ is finitely generated and $M_{\mathfrak{m}}=M_{\mathfrak{m}} \mathfrak{a}$. If $\mathfrak{a} \subset \mathfrak{m}$, then $\mathfrak{a} R_{\mathfrak{m}} \subset \operatorname{Jac}\left(R_{\mathfrak{m}}\right)$, which forces $M_{\mathfrak{m}}=0$ by Nakayama's Lemma. In this case any element of $M$ is annihilated by some element in $Z \backslash \mathfrak{m}$; since $M$ is finitely generated over $R$ and $Z$ is in the center of $R$ there exists $z \in Z \backslash \mathfrak{m}$ such that $M z=0$. Denote by $\mathfrak{b}$ the annihilator of $M$ in $Z$. We conclude that $\mathfrak{a}+\mathfrak{b}$ cannot be contained in any maximal ideal of $Z$, whence $\mathfrak{a}+\mathfrak{b}=Z$. It follows that $1-a \in \mathfrak{b}$ for some $a \in \mathfrak{a}$. If $M$ is faithful, we must have $a=1$.

Lemma 2.3. For any $r \in \mathbb{Q}, r \geq 0$, we have

$$
U_{r}(M)=\left\{\mathfrak{m} \in \operatorname{Max} Z \mid r_{P}(M) \leq r \text { for all } P \in \operatorname{Max}_{\mathfrak{m}} R\right\} .
$$

Proof. Let $\mathfrak{m} \in \operatorname{Max} Z$. Lemma 1.6(ii) together with Lemma 2.1 and the definition of $U_{r}(M)$ in section 1 show that $\mathfrak{m} \in U_{r}(M)$ if and only if $r_{P_{\mathfrak{m}}}\left(M_{\mathfrak{m}}\right) \leq r$ for all $P \in \operatorname{Max}_{\mathfrak{m}} R$. Since the image of $Z \backslash \mathfrak{m}$ in $R / P$ consists of invertible elements, we have $R_{\mathfrak{m}} / P_{\mathfrak{m}} \cong R / P$ and $M_{\mathfrak{m}} / M_{\mathfrak{m}} P_{\mathfrak{m}} \cong M / M P$, whence $r_{P_{\mathfrak{m}}}\left(M_{\mathfrak{m}}\right)=r_{P}(M)$.

Lemma 2.4. The supremum $r(M)=\sup \left\{r_{P}(M) \mid P \in \operatorname{Max} R\right\}$ is attained at some maximal ideal of $R$.

Proof. Note that $\operatorname{Max} Z \neq U_{s}(M)$ for any $s \in \mathbb{Q}$ such that $0 \leq s<r(M)$. Indeed, if $P \in \operatorname{Max} R$ satisfies $r_{P}(M)>s$, then $P \cap Z$ is a maximal ideal of $Z$ lying outside of $U_{s}(M)$ by Lemma 2.3. Since Max $Z$ is quasicompact and each $U_{s}(M)$ is open in $\operatorname{Max} Z$, we get

$$
\operatorname{Max} Z \neq \bigcup_{0 \leq s<r(M)} U_{s}(M) .
$$

Pick $\mathfrak{m} \in \operatorname{Max} Z$ contained in none of the subsets $U_{s}(M)$ with $s<r(M)$. Next, in the finite set $\operatorname{Max}_{\mathfrak{m}} R$ pick $P$ with the maximum value of $r_{P}(M)$. By Lemma 2.3 $r_{P}(M)>s$ for any $s \in \mathbb{Q}$ with $s<r(M)$. Hence $r_{P}(M)=r(M)$.

Lemma 2.5. Let $K$ be an ideal of $R$ such that $R / K$ is semilocal, and let $r=n / l$ for some integers $n \geq 0, l>0$. Suppose that $U_{r}(M)$ is quasicompact and $r_{Q}(M) \leq r$ for each $Q \in \operatorname{Max} R$ such that there exists $P \in \operatorname{Max} R$ satisfying $P \supset K$ and $P \cap Z=Q \cap Z$. Then:

(i) $I_{r}(M / M K)$ coincides with the image of $I_{r}(M)$ in $R / K$.

(ii) $I_{r}(M) \subset K$ if and only if $(M / M K)^{l} \cong(R / K)^{n}$ in $\mathcal{M}_{R}$.

(iii) If $I_{r}(M) \subset K$ then $r_{P}(M)=r$ for each $P \in \operatorname{Max} R$ with $P \supset K$.

Proof. If $\mathfrak{m}=P \cap Z$ where $P \in \operatorname{Max} R, P \supset K$, then we have $r_{Q}(M) \leq r$ for all $Q \in \operatorname{Max}_{\mathfrak{m}} R$ by the hypothesis; hence $\mathfrak{m} \in U_{r}(M)$ according to Lemma 2.3. The Remark following Lemma 1.10 now proves (i). Since $r_{P}(M) l \leq n$ for each $P \in \operatorname{Max} R$ with $P \supset K$, the $R / K$-module $(M / M K)^{l}$ is $n$-generated by Lemma 1.6. Hence (ii) follows from (i) and Lemma 1.4(ii). If $(M / M K)^{l} \cong(R / K)^{n}$, then $(M / M P)^{l} \cong(R / P)^{n}$ for any $P \in \operatorname{Max} R$ with $P \supset K$; the comparison of lengths of the two modules appearing in the latter isomorphism yields (iii).

Lemma 2.6. Suppose that there is an integer $n \geq 0$ such that $M_{\mathfrak{m}} \cong R_{\mathfrak{m}}^{n}$ in $\mathcal{M}_{R_{\mathfrak{m}}}$ for each $\mathfrak{m} \in \operatorname{Max} Z$. Then $M$ is projective; $M$ is a generator in $\mathcal{M}_{R}$ provided $M \neq 0$. For each $\mathfrak{m} \in \operatorname{Max} Z$ there exists $z \in Z \backslash \mathfrak{m}$ such that $M_{z} \cong R_{z}^{n}$ in $\mathcal{M}_{R_{z}}$. 
Proof. We first prove that $M$ is finitely presented. Consider any $\mathcal{M}_{R^{-}}$epimorphism $\varphi: R^{m} \rightarrow M$ with kernel $K$. We have to check that $K$ is finitely generated. For each $\mathfrak{m} \in \operatorname{Max} Z$ the localization $\varphi_{\mathfrak{m}}$ of $\varphi$ at $\mathfrak{m}$ gives rise to an exact sequence of $R_{\mathfrak{m}}$-modules $0 \rightarrow K_{\mathfrak{m}} \rightarrow R_{\mathfrak{m}}^{m} \rightarrow M_{\mathfrak{m}} \rightarrow 0$. Since $M_{\mathfrak{m}}$ is free, the sequence splits, and so $R_{\mathfrak{m}}^{m} \cong K_{\mathfrak{m}} \oplus R_{\mathfrak{m}}^{n}$. It follows that $m \geq n$ and $K_{\mathfrak{m}} \cong R_{\mathfrak{m}}^{m-n}$ by the cancellation property for projective modules over a semilocal ring [2, Ch. IV, (1.4)].

For any fixed $\mathfrak{m}$ we can pick $m$ elements $v_{1}, \ldots, v_{m} \in R^{m}$ with the property that $v_{n+1}, \ldots, v_{m} \in K$, the images of $v_{n+1}, \ldots, v_{m}$ in $K_{\mathfrak{m}}$ give a basis for $K_{\mathfrak{m}}$ over $R_{\mathfrak{m}}$, and the images of $v_{1}, \ldots, v_{n}$ in $R_{\mathfrak{m}}^{m}$ give a basis for a complementary summand. Let $K^{\prime}$ and $N$ be the submodules of $R^{m}$ generated by $v_{n+1}, \ldots, v_{m}$ and $v_{1}, \ldots, v_{n}$, respectively. Since $R_{\mathfrak{m}}^{m}=K_{\mathfrak{m}}^{\prime}+N_{\mathfrak{m}}$, there exists $z \in Z \backslash \mathfrak{m}$ such that $R^{m} z \subset K^{\prime}+N$. For any $\mathfrak{n} \in \operatorname{Max} Z$ with $z \notin \mathfrak{n}$ we have $R_{\mathfrak{n}}^{m}=K_{\mathfrak{n}}^{\prime}+N_{\mathfrak{n}}$. Since $K^{\prime} \subset K$, it follows that $\varphi_{\mathfrak{n}}\left(N_{\mathfrak{n}}\right)=M_{\mathfrak{n}}$. The $R_{\mathfrak{n}}$-module $N_{\mathfrak{n}}$ is generated by $n$ elements. Since the ring $R_{\mathfrak{n}}$ is weakly finite and $M_{\mathfrak{n}} \cong R_{\mathfrak{n}}^{n}$, the images of those elements in $M_{\mathfrak{n}}$ are a basis for $M_{\mathfrak{n}}$ over $R_{\mathfrak{n}}$. In other words, $\varphi_{\mathfrak{n}}$ induces an isomorphism of $N_{\mathfrak{n}}$ onto $M_{\mathfrak{n}}$. Hence $K_{\mathfrak{n}} \cap N_{\mathfrak{n}}=0$, and therefore $K_{\mathfrak{n}}=K_{\mathfrak{n}}^{\prime}$.

Denote by $\mathcal{U}$ the collection of all open subsets $U$ of Max $Z$ with the property that there exists a finitely generated submodule $L \subset K$, depending on $U$, such that $K_{\mathfrak{n}}=L_{\mathfrak{n}}$ for all $\mathfrak{n} \in U$. We have just proved that each $\mathfrak{m} \in \operatorname{Max} Z$ has an open neighborhood contained in $\mathcal{U}$. It is also clear that $U \cup U^{\prime} \in \mathcal{U}$ whenever $U, U^{\prime} \in \mathcal{U}$. Since the space $\operatorname{Max} Z$ is quasicompact, we conclude that $\operatorname{Max} Z \in \mathcal{U}$. This means that there exists a finitely generated submodule $L \subset K$ such that $K_{\mathfrak{n}}=L_{\mathfrak{n}}$ for all $\mathfrak{n} \in \operatorname{Max} Z$. But then $K=L$. Thus $M$ is finitely presented, as claimed.

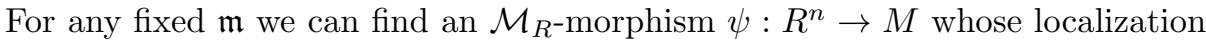
$R_{\mathfrak{m}}^{n} \rightarrow M_{\mathfrak{m}}$ is an isomorphism. Since $M$ is finitely generated, there exists $s \in Z \backslash \mathfrak{m}$ such that the $\mathcal{M}_{R_{s}}$-morphism $\psi_{s}: R_{s}^{n} \rightarrow M_{s}$ induced by $\psi$ is surjective. Since the $R_{s}$-module $M_{s}$ is finitely presented, $\operatorname{Ker} \psi_{s}$ is finitely generated. Then $\operatorname{Ker} \psi_{s}$ is annihilated by some element $z \in Z \backslash \mathfrak{m}$. We may assume that $Z_{z}$ is a localization of $Z_{s}$, in which case $\psi_{s}$ induces an isomorphism $R_{z}^{n} \rightarrow M_{z}$.

For $V \in \mathcal{M}_{R}$ and $\mathfrak{m} \in \operatorname{Max} Z$ the canonical map

$$
\operatorname{Hom}_{R}(M, V) \otimes_{Z} Z_{\mathfrak{m}} \rightarrow \operatorname{Hom}_{R_{\mathfrak{m}}}\left(M_{\mathfrak{m}}, V_{\mathfrak{m}}\right)
$$

is bijective by [2, Ch. III, (4.5)]. Let now $\xi: \operatorname{Hom}_{R}(M, V) \rightarrow \operatorname{Hom}_{R}(M, W)$ be the map induced by an $\mathcal{M}_{R^{-}}$epimorphism $V \rightarrow W$. Since $M_{\mathfrak{m}}$ is projective in $\mathcal{M}_{R_{\mathfrak{m}}}$, the map $\xi \otimes \mathrm{id}: \operatorname{Hom}_{R}(M, V) \otimes_{Z} Z_{\mathfrak{m}} \rightarrow \operatorname{Hom}_{R}(M, W) \otimes_{Z} Z_{\mathfrak{m}}$ is surjective for each $\mathfrak{m}$, but then $\xi$ is itself surjective. This proves that $M$ is projective in $\mathcal{M}_{R}$.

Suppose that $M \neq 0$. Then $n \neq 0$, and so $M_{\mathfrak{m}} \neq 0$ for each $\mathfrak{m} \in \operatorname{Max} Z$. Denote by $T$ the trace ideal of $M$. Thus $T=\sum f(M)$ where $f$ runs over $\operatorname{Hom}_{R}(M, R)$. Since $\operatorname{Hom}_{R_{\mathfrak{m}}}\left(M_{\mathfrak{m}}, R_{\mathfrak{m}}\right) \cong \operatorname{Hom}_{R}(M, R) \otimes_{Z} Z_{\mathfrak{m}}$, the trace ideal of the $R_{\mathfrak{m}}$-module $M_{\mathfrak{m}}$ coincides with $T_{\mathfrak{m}}$. It follows that $T_{\mathfrak{m}}=R_{\mathfrak{m}}$ since $M_{\mathfrak{m}}$ is free. As this is valid for each $\mathfrak{m}$, we get $T=R$. This means that $M$ is a generator.

Lemma 2.7. Let $P \in \operatorname{Spec} R$ and $\mathfrak{m} \in \operatorname{Max} Z$. If $P \cap Z \subset \mathfrak{m}$ then $P \subset Q$ for some $Q \in \operatorname{Max}_{\mathfrak{m}} R$.

Proof. The hypothesis implies that $1 \notin P_{\mathfrak{m}}$. So $P_{\mathfrak{m}}$ is contained in a maximal ideal of $R_{\mathfrak{m}}$, that is, an ideal $Q_{\mathfrak{m}}$ for some $Q \in \operatorname{Max}_{\mathfrak{m}} R$. Then $P \subset Q$.

Recall that a Jacobson ring is a ring in which every prime ideal is an intersection of primitive ideals. 
Lemma 2.8. Let $P \in \operatorname{Spec} R$ and $\mathfrak{p}=P \cap Z$. Suppose that $Z$ is a Jacobson ring and $R_{\mathfrak{p}} / \mathfrak{p} R_{\mathfrak{p}}$ is an artinian ring. Then for any $z \in Z \backslash \mathfrak{p}$ the intersection $K$ of all ideals $Q \in \operatorname{Max} R$ such that $P \subset Q$ and $z \notin Q$ coincides with $P$.

Proof. Clearly $P \subset K$. In view of Lemma $2.7 K \cap Z$ coincides with the intersection $\mathfrak{b}$ of all ideals $\mathfrak{m} \in \operatorname{Max} Z$ such that $\mathfrak{p} \subset \mathfrak{m}$ and $z \notin \mathfrak{m}$. Since $Z$ is a Jacobson ring, we have $\mathfrak{p}=\mathfrak{a} \cap \mathfrak{b}$ where $\mathfrak{a}$ denotes the intersection of all ideals $\mathfrak{m} \in \operatorname{Max} Z$ such that $\mathfrak{p} \subset \mathfrak{m}$ and $z \in \mathfrak{m}$. In particular, $\mathfrak{a} \mathfrak{b} \subset \mathfrak{p}$. Since $z \in \mathfrak{a}$, we have $\mathfrak{a} \not \subset \mathfrak{p}$; hence $\mathfrak{b} \subset \mathfrak{p}$ because $\mathfrak{p}$ is prime. We conclude that $K \cap Z=\mathfrak{p}$.

Let $R^{\prime}=R_{\mathfrak{p}} / P_{\mathfrak{p}} \cong R / P \otimes_{Z} Z_{\mathfrak{p}}$. Since $R / P$ is a prime ring, its central subring $Z / \mathfrak{p}$ contains no zero divisors of $R / P$ other than 0 . Hence $R / P$ is embedded into $R^{\prime}$. The ring $R^{\prime}$, as a homomorphic image of $R_{\mathfrak{p}} / \mathfrak{p} R_{\mathfrak{p}}$, is artinian. Each nonzero ideal of $R^{\prime}$ intersects $R / P$ nontrivially. It follows that $R^{\prime}$ is prime, in which case $R^{\prime}$ is actually simple. If the ideal $K^{\prime}$ of $R^{\prime}$ generated by the image of $K$ contained $1, K / P$ would have a nonzero intersection with $Z / \mathfrak{p}$, which is impossible. We must have $K^{\prime}=0$, which entails $K / P=0$, i.e., $K=P$.

Remark. If $R$ is module-finite over $Z$, then $R_{\mathfrak{p}} / \mathfrak{p} R_{\mathfrak{p}}$ is a finite dimensional algebra over a field, so that the artinian hypothesis in Lemma 2.8 is fulfilled. In this case $\mathfrak{p} R_{\mathfrak{p}} \subset \operatorname{Jac}\left(R_{\mathfrak{p}}\right)$ by [1, Corollary to Lemma 2] or [9, Lemma 3.1], which implies that $R_{\mathfrak{p}}$ is semilocal.

It is well-known that the Jacobson property goes up from $Z$ to $R$ in the modulefinite case. The first result of this kind, due to Curtis [9, Th. 4.3], assumed ACC on $Z$-submodules of $R$. Subsequently several generalizations have been found, e.g. [8], [23], [25]. Under previous assumptions $R_{z}$ is module-finite over $Z_{z}$ and $Z_{z}$ is Jacobson by [4, Ch. V, $\S 3$, Th. 3]; so $R_{z}$ is Jacobson for any $z \in Z$. This reduces to the conclusion of Lemma 2.8 .

\section{Semilocal factor algebras of module algebras}

Suppose that $H$ is a Hopf algebra and $A$ a left $H$-module algebra over the ground field $k$. The compatibility of the $H$-module structure with the algebra structure on $A$ is expressed by means of the identities

$$
h 1_{A}=\varepsilon(h) 1_{A}, \quad h(a b)=\sum\left(h_{(1)} a\right)\left(h_{(2)} b\right)
$$

where $h \in H, a, b \in A$, and $1_{A}$ is the unity element of $A$. For an ideal $I$ of $A$ and a subcoalgebra $C$ of $H$ put

$$
I_{C}=\{a \in A \mid C a \subset I\} .
$$

Clearly $I_{C}$ is also an ideal of $A$. In particular, $I_{H}$ is the largest $H$-stable ideal of $A$ contained in $I$. If $C, C^{\prime}$ are two subcoalgebras with $C \subset C^{\prime}$ then $I_{C} \supset I_{C^{\prime}}$.

Our subsequent arguments require the factor algebras $A / I_{C}$ to be semilocal. We wish to know the cases in which this property of $A / I_{C}$ can be established.

We always consider $\operatorname{Hom}(C, A / I)$ equipped with the convolution multiplication. For $a \in A$ define $\tilde{a} \in \operatorname{Hom}(C, A / I)$ by the rule

$$
\tilde{a}(c)=c a+I, \quad c \in C .
$$

The map $\tau: A \rightarrow \operatorname{Hom}(C, A / I)$ given by the assignment $a \mapsto \tilde{a}$ is a homomorphism of algebras and $\operatorname{Ker} \tau=I_{C}$. The inclusion $k \hookrightarrow A / I$ allows us to identify the dual algebra $C^{*}$ of $C$ with a subalgebra of $\operatorname{Hom}(C, A / I)$. 
Lemma 3.1. If $\operatorname{dim} C<\infty$, then $\operatorname{Hom}(C, A / I)=\tau(A) C^{*}$ and so $\operatorname{Hom}(C, A / I)$ is left module-finite over $\tau(A)$.

Proof. The verification is straightforward. In case $I=0$ the statement is contained in [29, Lemma 2.1(iv)]. The general case is immediate since the canonical projection $A \rightarrow A / I$ induces a surjective algebra homomorphism $\operatorname{Hom}(C, A) \rightarrow \operatorname{Hom}(C, A / I)$.

Remark. It is probably not true that $\operatorname{Hom}(C, A / I)$ is right module-finite over $\tau(A)$ in case of Hopf algebras whose antipode is not bijective. This is essentially the reason for our use of left side conditions in this section.

Lemma 3.2. If $C$ and $A / I$ are finite dimensional, then so too is $A / I_{C}$.

This is clear since $A / I_{C}$ is embedded into $\operatorname{Hom}(C, A / I)$.

Lemma 3.3. Let $T$ be a ring left module-finite over a subring $R$. Suppose that $R$ is either (a) left noetherian or (b) left module-finite over a commutative subring $R^{\prime}$. If $T$ is left artinian (semiprimary, semilocal), then so too is $R$.

Proof. (i) If $T$ is left artinian then $R$ is left artinian by Björk's results. In case (a) [3, Cor. 0.2] applies. In case (b) $T$ is left module-finite over $R^{\prime}$; so $R^{\prime}$ is artinian by [3, Th. 3.3]. Then $T$ has finite length as an $R^{\prime}$-module with respect to left multiplications, and the same holds for $R$.

(ii) Suppose that $T$ is semiprimary and $J=\operatorname{Jac}(T)$. Part (i) shows that the subring $R /(J \cap R)$ of the artinian ring $T / J$ is left artinian. Since $J \cap R$ is a nilpotent ideal of $R$, it is clear that $R$ is semiprimary.

(iii) A result of Camps and Dicks [6] says that a subring of a semilocal ring is itself semilocal provided that the subring is full, that is, each non-invertible element of the subring is not invertible in the ambient ring. We will check that $R$ is a full subring of $T$; it will follow then that $R$ is semilocal whenever so is $T$. Let $x \in R$ be invertible in $T$. We have to show that $x^{-1} \in R$. In case (a) $T$ is a noetherian $R$-module on the left side. Hence the chain of submodules $R \subset R x^{-1} \subset R x^{-2} \subset \cdots$ is ultimately constant, i.e., $x^{-n} \in R x^{1-n}$ for some $n>0$. Multiplying by $x^{n-1}$ proves the claim.

In case (b) $T$ is a finitely generated $R^{\prime}$-module on the left side. The right multiplication by $x^{-1}$ defines an endomorphism $f$ of that module. Since $R^{\prime}$ is commutative, $f$ satisfies an equation $f^{n}=\sum_{i=0}^{n-1} c_{i} f^{i}$ for some $n>0$ and $c_{0}, \ldots, c_{n-1} \in R^{\prime}$. Then we have $t x^{-n}=\sum_{i=0}^{n-1} c_{i} t x^{-i}$ for all $t \in T$. Substituting $t=1$, we deduce that $x^{-n} \in \sum_{i=0}^{n-1} R^{\prime} x^{-i} \subset R x^{1-n}$, which leads to the desired conclusion.

Lemma 3.4. Suppose that $\operatorname{dim} C<\infty$ and $A$ is either left noetherian or left modulefinite over a commutative subring. If $A / I$ is left artinian (semiprimary, semilocal), then so too is $A / I_{C}$.

Proof. We take $T=\operatorname{Hom}(C, A / I)$ and $R=\tau(A)$. By Lemma $3.1 T$ is left modulefinite over $R$. Let us identify $T$ with the algebra $C^{*} \otimes A / I$ by means of the canonical isomorphism. Thus $T$ is left module-finite over the subring $1 \otimes A / I$ isomorphic to $A / I$. If $A / I$ is left artinian, so is $T$. Since the finite dimensional subalgebra $C^{*} \otimes 1$ centralizers $1 \otimes A / I$, the ideal $J=C^{*} \otimes \operatorname{Jac}(A / I)$ of $T$ is contained in the Jacobson radical of $T$ [15, Prop. 5.7]. If $A / I$ is semilocal, then $T / J$ is artinian, and it follows that $T$ is semilocal. If $A / I$ is semiprimary then $J$ is nilpotent; hence $T$ is 
semiprimary. Since $A / I_{C} \cong \tau(A)$, an application of Lemma 3.3 yields all conclusions of Lemma 3.4.

Remark. The conclusion of Lemma 3.4 can be rephrased in the language of [20, Def. 3.4] as follows: the action of $H$ on $A$ is $\mathcal{F}$-continuous where $\mathcal{F}$ is the filter consisting of those ideals $I$ of $A$ for which $A / I$ is left artinian in one case, semiprimary in the second and semilocal in the third. Another result of this kind will be presented in Lemma 3.6.

Lemma 3.5. Let $Z \subset R \subset T$ be a tower of rings where $Z$ is central in $R$, the ring $R$ has semilocal localizations with respect to $Z$ and $T$ is left module-finite over $R$. If $T$ is semilocal then $R$ is semilocal. If $T$ is semiprimary then so too is $Z$; if also all rings $R_{\mathfrak{m}} / \mathfrak{m} R_{\mathfrak{m}}$ with $\mathfrak{m} \in \operatorname{Max} Z$ are semiprimary then $R$ is semiprimary.

Proof. Note that the version of Lemma 2.2 for left $R$-modules is also valid since we may replace $R$ with the opposite ring. Take $M=T$ regarded as an $R$-module with respect to left multiplications. Lemma 2.2 shows that the equality $a T=T$ for $a \in Z$ implies $a Z=Z$. In other words, $a^{-1} \in Z$ whenever $a$ is invertible in $T$. So $Z$ is a full subring of $T$, and the Camps-Dicks Theorem ensures that $Z$ is semilocal (cf. the proof of Lemma 3.3). Since the set Max $Z$ is finite, by Lemma 2.1 $R$ has finitely many right primitive factor algebras $R / P$, and each of those is simple artinian. Hence $R$ is semilocal.

Suppose that $T$ is semiprimary. Since $J=\operatorname{Jac}(T)$ is nilpotent, the ring $R$ (resp., $Z$ ) is semiprimary if and only if so is $R /(R \cap J)$ (resp., $Z /(Z \cap J)$ ). Passing to the tower of rings $Z /(Z \cap J) \subset R /(R \cap J) \subset T / J$, we may assume that $T$ is artinian. Given $a \in Z$, there exists an integer $n>0$ such that $a^{n} T=a^{n+1} T$. Now $a^{n} T$ is a finitely generated left $R$-submodule of $T$ since $a R=R a$. Applying Lemma 2.2 with $M=a^{n} T$ and $\mathfrak{a}=a Z$, we deduce that $(1-b) a^{n} T=0$ for some $b \in a Z$. If $a \in \operatorname{Jac}(Z)$, then $1-b$ is invertible, whence $a^{n} T=0$, i.e., $a^{n}=0$. This $\operatorname{shows}$ that $\operatorname{Jac}(Z)$ is nil. Since nil subrings of artinian rings are nilpotent, $\operatorname{Jac}(Z)$ is nilpotent. This means that $Z$ is semiprimary. Since $Z$ is commutative, $Z$ is the finite direct product of local rings $Z_{\mathfrak{m}}, \mathfrak{m} \in \operatorname{Max} Z$, with nilpotent maximal ideals $\mathfrak{m} Z_{\mathfrak{m}}$. Then $R \cong \prod R_{\mathfrak{m}}$ and $\mathfrak{m} R_{\mathfrak{m}}$ is a nilpotent ideal of $R_{\mathfrak{m}}$ for each $\mathfrak{m}$. If $R_{\mathfrak{m}} / \mathfrak{m} R_{\mathfrak{m}}$ is semiprimary, so too is $R_{\mathfrak{m}}$. When all rings $R_{\mathfrak{m}}$ are semiprimary, $R$ is semiprimary.

Lemma 3.6. Suppose that $\operatorname{dim} C<\infty$ and $A$ has semilocal localizations with respect to a central subring $Z$.

(i) If $A / I$ is semilocal then $A / I_{C}$ is semilocal.

(ii) If $A / I$ is semiprimary then $Z /\left(Z \cap I_{C}\right)$ is semiprimary.

(iii) If $A / I$ and all rings $A_{\mathfrak{m}} / \mathfrak{m} A_{\mathfrak{m}}$ are semiprimary then $A / I_{C}$ is semiprimary.

Proof. We apply Lemma 3.5 to the tower $\tau(Z) \subset \tau(A) \subset \operatorname{Hom}(C, A / I)$. As pointed out in the proof of Lemma 3.4, $\operatorname{Hom}(C, A / I)$ is semilocal (semiprimary) whenever so is $A / I$.

\section{The orbit relation on the maximal spectrum}

We continue to assume that $A$ is an $H$-module algebra. For $P, Q \in \operatorname{Max} A$ define $P \leq_{H} Q$ if $P_{C} \subset Q$ for some finite dimensional subcoalgebra $C \subset H$. Here $P_{C}$ denotes the ideal of $A$ defined in section 3 . 
Lemma 4.1. The relation $\leq_{H}$ is reflexive and transitive.

Proof. If $C=k$, then $P_{C}=P$. Since $P \subset P$, we get $P \leq_{H} P$. Suppose that $P, P^{\prime}, P^{\prime \prime} \in \operatorname{Max} A$ satisfy $P \leq_{H} P^{\prime}$ and $P^{\prime} \leq_{H} P^{\prime \prime}$. Then $P_{C} \subset P^{\prime}$ and $P_{C^{\prime}}^{\prime} \subset P^{\prime \prime}$ for some finite dimensional subcoalgebras $C, C^{\prime} \subset H$. Note that $C C^{\prime}$ is also a finite dimensional subcoalgebra of $H$. If $a \in P_{C C^{\prime}}$, that is, $C C^{\prime} a \subset P$, then $C^{\prime} a \subset P_{C} \subset$ $P^{\prime}$, whence $a \in P_{C^{\prime}}^{\prime} \subset P^{\prime \prime}$. This shows that $P_{C C^{\prime}} \subset P^{\prime \prime}$, and therefore $P \leq_{H} P^{\prime \prime}$.

If $H=k G$ is a group algebra, then any finite dimensional subcoalgebra $C$ of $H$ is spanned by a finite subset, say $X$, of $G$. Clearly $P_{C}=\bigcap_{g \in X} g^{-1}(P)$. If $Q \in \operatorname{Max} A$ contains $P_{C}$, then $Q$ contains the product of the ideals $g^{-1}(P), g \in X$, taken in any order; since $Q$ is prime, $Q \supset g^{-1}(P)$ for some $g \in X$. The maximality of $P$ ensures then that $Q=g^{-1}(P)$. Thus $P \leq_{H} Q$ if and only if $P$ and $Q$ lie in the same $G$-orbit.

The previous example suggests that $\leq_{H}$ may also be symmetric, that is, an equivalence relation on $\operatorname{Max} A$ in general. It is not clear whether this is always true. We will be able to provide a confirmation in several cases. When the relation $\leq_{H}$ is symmetric, we call it the $H$-orbit equivalence relation.

Note that $P_{H}$ coincides with the intersection of the family of ideals $P_{C}$ with $C$ a finite dimensional subcoalgebra. It follows that $P_{H} \subset Q_{H}$ whenever $P \leq_{H} Q$. If $P \leq_{H} Q$ and $Q \leq_{H} P$ then $P_{H}=Q_{H}$, that is, $P$ and $Q$ belong to the same $H$-stratum, in the language of [5]. In general the $H$-stratification defines a coarser equivalence relation.

The proof of the next lemma uses essentially the same argument as given by Chin [7, Lemma 2.2] in the case where $H$ is finite dimensional and pointed; it was further generalized by Montgomery and Schneider [21, Th. 3.7].

Lemma 4.2. Suppose that $H^{\prime}$ is a Hopf subalgebra of $H$ containing the coradical of $H$. Then for $P, Q \in \operatorname{Max} A$ one has $P \leq_{H} Q$ if and only if $P \leq_{H^{\prime}} Q$. The relation $\leq_{H}$ is symmetric if and only if so is $\leq_{H^{\prime}}$.

Proof. Suppose that $C$ is a finite dimensional subcoalgebra of $H$ and $C_{0}$ denotes the coradical of $C$. Consider the coradical filtration $C_{0} \subset C_{1} \subset \cdots$ of $C$. As $\operatorname{dim} C<\infty$, we have $C_{n}=C$ for some $n$. Let $P \in \operatorname{Max} A$. We will prove by induction on $i \geq 0$ that $P_{C_{0}}^{i+1} \subset P_{C_{i}}$. For $i=0$ this is clear. Suppose that the claim is valid for some $i \geq 0$ and $c \in C_{i+1}$. Since $\Delta(c) \in C_{0} \otimes C+C \otimes C_{i}$, we deduce

$$
c\left(P_{C_{0}}^{i+2}\right) \subset \sum_{(c)} c_{(1)}\left(P_{C_{0}}\right) \cdot c_{(2)}\left(P_{C_{0}}^{i+1}\right) \subset P
$$

showing that $P_{C_{0}}^{i+2} \subset P_{C_{i+1}}$. In particular, $P_{C_{0}}^{n+1} \subset P_{C}$. It follows that for $Q \in \operatorname{Max} A$ the inclusions $P_{C} \subset Q$ and $P_{C_{0}} \subset Q$ are equivalent to each other. Since $C_{0} \subset H^{\prime}$, we conclude that $P \leq_{H} Q$ if and only if $P \leq_{H^{\prime}} Q$.

Corollary 4.3. If $H$ is pointed with the group $G$ of grouplike elements then $P \leq_{H} Q$ for $P, Q \in \operatorname{Max} A$ if and only if $P$ and $Q$ lie in the same $G$-orbit.

Proof. In this case the coradical of $H$ coincides with the group algebra $k G$.

Proposition 4.4. If $A$ is right artinian then $\leq_{H}$ is symmetric. 
Proof. If $C$ and $C^{\prime}$ are two finite dimensional subcoalgebras of $H$, so also is $C+C^{\prime}$, and $P_{C+C^{\prime}}=P_{C} \cap P_{C^{\prime}}$. Since $A$ satisfies DCC on right ideals, the set of ideals $P_{C}$ with $C$ a finite dimensional subcoalgebra of $H$, contains a smallest element which has to coincide with $P_{H}$. Hence for $P, Q \in \operatorname{Max} A$ one has $P \leq_{H} Q$ if and only if $P_{H} \subset Q$. The right artinian $H$-module algebra $A / P_{H}$ has a maximal ideal $P / P_{H}$ which contains no nonzero $H$-stable ideals of $A / P_{H}$. By [29, Lemma 4.2] $A / P_{H}$ is $H$-simple. If $P_{H} \subset Q$, then $Q_{H}$ is an $H$-stable ideal of $A$ containing $P_{H}$, and we must have $Q_{H}=P_{H}$. The inclusion $Q_{H} \subset P$ entails $Q \leq_{H} P$.

Proposition 4.5. Suppose that $X \subset \operatorname{Max} A$ is a subset such that for each $P \in X$ and each finite dimensional subcoalgebra $C$ of $H$ the factor ring $A / P_{C}$ is semiprimary and each maximal ideal of $A$ containing $P_{C}$ lies in $X$. If either (a) $\operatorname{dim} H<\infty$ or (b) $H$ is generated by a family $\mathcal{H}$ of Hopf subalgebras such that the relation $\leq_{H^{\prime}}$ is symmetric on $X$ for each $H^{\prime} \in \mathcal{H}$, then the relation $\leq_{H}$ is symmetric on $X$.

Proof. In case (a) $P_{H}$ is the smallest element in the set of ideals $P_{C}$ with $C$ a finite dimensional subcoalgebra. Hence for $P, Q \in \operatorname{Max} A$ one has $P \leq_{H} Q$ if and only if $P_{H} \subset Q$. The $H$-module algebra $A / P_{H}$ is semiprimary by the hypothesis. Its maximal ideal $P / P_{H}$ contains no nonzero $H$-stable ideals of $A / P_{H}$. It follows that $A / P_{H}$ is $H$-semiprime, i.e., $A / P_{H}$ has no nonzero $H$-stable nilpotent ideals. By $[29$, Th. 0.3 and Lemma 4.2] $A / P_{H}$ is $H$-simple. Then the inclusion $P_{H} \subset Q$ implies $P_{H}=Q_{H}$, and so $Q \leq_{H} P$.

Assume now that $H$ satisfies condition (b). Denote by $\mathcal{C}$ the collection of subcoalgebras $C$ of $H$ such that $\operatorname{dim} C<\infty$ and for any pair $P, Q \in X$ satisfying $P_{C} \subset Q$ one has $Q \leq_{H} P$. By the hypothesis $\mathcal{C}$ contains all finite dimensional subcoalgebras of any $H^{\prime} \in \mathcal{H}$.

We claim that $C+C^{\prime} \in \mathcal{C}$ and $C C^{\prime} \in \mathcal{C}$ whenever $C, C^{\prime}$ are both from $\mathcal{C}$. Suppose that $P, Q \in X$ are such that $P_{C+C^{\prime}} \subset Q$. Since $P_{C+C^{\prime}}=P_{C} \cap P_{C^{\prime}} \supset P_{C} P_{C^{\prime}}$ and $Q$ is a prime ideal, we have either $P_{C} \subset Q$ or $P_{C^{\prime}} \subset Q$, whence $Q \leq_{H} P$. This proves the first inclusion in our claim.

We also have to show that $Q \leq_{H} P$ whenever $P, Q \in X$ satisfy $P_{C C^{\prime}} \subset Q$. Denote by $Y$ the set of maximal ideals of $A$ containing $P_{C}$. By the hypothesis $Y \subset X$, and $Y$ is finite since $A / P_{C}$ is semiprimary. If $J$ denotes the intersection of all ideals from $Y$, then $J / P_{C}$ coincides with the Jacobson radical of $A / P_{C}$, which is nilpotent. It follows that there exists a finite sequence $Q_{1}, \ldots, Q_{n}$ of ideals from $Y$ (with repetitions allowed) such that $Q_{1} \cdots Q_{n} \subset P_{C}$. If $a_{1}, \ldots, a_{n} \in A$ are any elements such that $C^{\prime} a_{i} \subset Q_{i}$ for each $i=1, \ldots, n$, then $C^{\prime}\left(a_{1} \cdots a_{n}\right) \subset Q_{1} \cdots Q_{n} \subset P_{C}$, and then $C C^{\prime}\left(a_{1} \cdots a_{n}\right) \subset P$. This shows that $\left(Q_{1}\right)_{C^{\prime}} \cdots\left(Q_{n}\right)_{C^{\prime}} \subset P_{C C^{\prime}} \subset Q$. Since $Q$ is prime, we must have $\left(Q_{i}\right)_{C^{\prime}} \subset Q$ for at least one $i$. The inclusions $C^{\prime}, C \in \mathcal{C}$ imply that $Q \leq_{H} Q_{i}$ and $Q_{i} \leq_{H} P$. The transitivity of the relation $\leq_{H}$ entails $Q \leq_{H} P$, as required.

It is clear now that the union $U$ of all coalgebras from $\mathcal{C}$ is a subalgebra of $H$. If $H^{\prime} \in \mathcal{H}$ then $H^{\prime} \subset U$ since $H^{\prime}$ is the union of its finite dimensional subcoalgebras. Since $H$ is generated by $\mathcal{H}$, we get $U=H$. Each finite dimensional subcoalgebra $C$ of $H$ is contained therefore in some $C^{\prime} \in \mathcal{C}$; since $P_{C} \supset P_{C^{\prime}}$ for any $P \in X$, it is clear that $C \in \mathcal{C}$. Thus $Q \leq_{H} P$ whenever $P, Q \in X$ satisfy $P_{C} \subset Q$.

There is a different interpretation of the relation $P \leq_{H} Q$ in terms of certain operations with modules. Denote by $\mathcal{M}^{H}$ the category of right $H$-comodules. Given $U \in \mathcal{M}^{H}$ and $V \in \mathcal{M}_{A}$, we define right $A$-module structures on vector spaces $U \otimes V$ 
and $\operatorname{Hom}(U, V)$ by the rules

$$
\begin{aligned}
(u \otimes v) a & =\sum u_{(0)} \otimes v\left(\left(S u_{(1)}\right) a\right), \\
(\eta a)(u) & =\sum \eta\left(u_{(0)}\right)\left(u_{(1)} a\right)
\end{aligned}
$$

where $u \in U, v \in V, a \in A, \eta \in \operatorname{Hom}(U, V)$ and $S: H \rightarrow H$ is the antipode (see [29, section 1]). If $\operatorname{dim} U<\infty$ then $U^{*}$ is a right $H$-comodule with structure map $U^{*} \rightarrow U^{*} \otimes H, \xi \mapsto \sum \xi_{(0)} \otimes \xi_{(1)}$, such that

$$
\sum \xi_{(0)}(u) \xi_{(1)}=\sum \xi\left(u_{(0)}\right) S u_{(1)}
$$

for all $u \in U$. Note that the evaluation map ev : $U^{*} \otimes U \rightarrow k$ is an $\mathcal{M}^{H}$-morphism provided $k$ has the trivial comodule structure.

Lemma 4.6. Let $U \in \mathcal{M}^{H}$ and $V, W \in \mathcal{M}_{A}$.

(i) $\operatorname{Hom}_{A}(U \otimes V, W) \cong \operatorname{Hom}_{A}(V, \operatorname{Hom}(U, W))$.

(ii) If $\operatorname{dim} U<\infty$ then $U \otimes V \cong \operatorname{Hom}\left(U^{*}, V\right)$ in $\mathcal{M}_{A}$.

Proof. (i) This is the isomorphism from [29, Lemma 1.1]. It is induced by the canonical linear bijection $\operatorname{Hom}(U \otimes V, W) \cong \operatorname{Hom}(V, \operatorname{Hom}(U, W))$.

(ii) We obtain $\operatorname{Hom}_{A}\left(U^{*} \otimes(U \otimes V), V\right) \cong \operatorname{Hom}_{A}\left(U \otimes V, \operatorname{Hom}\left(U^{*}, V\right)\right)$ as a special case of (i). The canonical map $\varphi: U \otimes V \rightarrow \operatorname{Hom}\left(U^{*}, V\right)$ corresponds to the composite

$$
U^{*} \otimes(U \otimes V) \cong\left(U^{*} \otimes U\right) \otimes V \stackrel{\text { ev } \otimes \mathrm{id}}{\longrightarrow} k \otimes V \cong V .
$$

Since the latter is an $\mathcal{M}_{A}$-morphism by functoriality, so too is $\varphi$. The assumption $\operatorname{dim} U<\infty$ entails the bijectivity of $\varphi$. Thus $\varphi$ is an isomorphism in $\mathcal{M}_{A}$.

Lemma 4.7. Suppose that $A / P$ and $A / Q$ are simple artinian. Let $V$ and $W$ be simple right $A$-modules whose annihilators coincide with $P$ and $Q$, respectively.

(i) $P \leq_{H} Q$ if and only if $W$ is a subfactor of the right $A$-module $\operatorname{Hom}(U, V)$ for some finite dimensional $U \in \mathcal{M}^{H}$.

(ii) If $W$ is a submodule of $\operatorname{Hom}(U, V)$ then both $P \leq_{H} Q$ and $Q \leq_{H} P$ hold.

(iii) If $W$ is a factor module of $\operatorname{Hom}(U, V)$ and the antipode of $H$ is bijective then $P \leq_{H} Q$ and $Q \leq_{H} P$ too.

Proof. (i) There is an isomorphism $A / P \cong V^{n}$ in $\mathcal{M}_{A}$ for some integer $n>0$. Suppose that there exists a finite dimensional subcoalgebra $C$ of $H$ such that $P_{C} \subset Q$. We may regard $C$ as a right $H$-comodule with respect to the comultiplication. The right $A$-module structure on $\operatorname{Hom}(C, A / P)$ derives from the algebra homomorphism $\tau: A \rightarrow \operatorname{Hom}(C, A / P)$ defined in section 3. Since $\operatorname{Ker} \tau=P_{C}$, the factor algebra $A / P_{C}$ is embedded in $\operatorname{Hom}(C, A / P)$. As $W$ is a simple $A / P_{C}$-module, $W$ is a subfactor of $\operatorname{Hom}(C, A / P)$ as a right $A$-module. The latter module is the direct sum of $n$ copies of $\operatorname{Hom}(C, V)$. Hence $W$ is a subfactor of $\operatorname{Hom}(C, V)$.

Conversely, suppose that $W$ is a subfactor of $\operatorname{Hom}(U, V)$ for some finite dimensional $U \in \mathcal{M}^{H}$. Since $H$ is an injective cogenerator in $\mathcal{M}^{H}$, there exists a monomorphism $\varphi: U \rightarrow H^{m}$ in $\mathcal{M}^{H}$ for some integer $m>0$. Then $\varphi(U) \subset C^{m}$ for a suitable finite dimensional subcoalgebra $C \subset H$. Hence $\operatorname{Hom}(U, V)$ is a homomorphic image of the right $A$-module $\operatorname{Hom}\left(C^{m}, V\right)$. It follows that $W$ is a subfactor of 
$\operatorname{Hom}(C, V)$, and also of $\operatorname{Hom}(C, A / P)$. Using again the equality $\operatorname{Ker} \tau=P_{C}$ from the previous paragraph, we deduce that $P_{C}$ annihilates $W$, whence $P_{C} \subset Q$.

(ii) If there exists an $\mathcal{M}_{A}$-monomorphism $W \rightarrow \operatorname{Hom}(U, V)$ then there also exists a nonzero $\mathcal{M}_{A}$-morphism $U \otimes W \rightarrow V$ by Lemma 4.6(i). The latter has to be surjective since $V$ is simple. Lemma 4.6(ii) shows that $V$ is a subfactor of $\operatorname{Hom}\left(U^{*}, W\right)$, whence $Q \leq_{H} P$ by part (i).

(iii) Suppose that the antipode is bijective. Then any $U \in \mathcal{M}^{H}, \operatorname{dim} U<\infty$, is isomorphic to $\left(U^{\prime}\right)^{*}$ for some finite dimensional $U^{\prime} \in \mathcal{M}^{H}$. By Lemma 4.6(ii) $\operatorname{Hom}(U, V) \cong U^{\prime} \otimes V$. If there exists an $\mathcal{M}_{A}$-epimorphism $\operatorname{Hom}(U, V) \rightarrow W$ then there also exists a nonzero $\mathcal{M}_{A}$-morphism $V \rightarrow \operatorname{Hom}\left(U^{\prime}, W\right)$ by Lemma 4.6(i). In this case $V$ is isomorphic with a submodule of $\operatorname{Hom}\left(U^{\prime}, W\right)$, whence $Q \leq_{H} P$.

Remark. We may regard $\mathcal{M}_{A}$ as a right module category over the tensor category $\left(\mathcal{M}^{H}\right)^{\text {op }}$, opposite to $\mathcal{M}^{H}$, with respect to the bifunctor $(V, U) \mapsto \operatorname{Hom}(U, V)$. Lemma 4.7 shows that $\leq_{H}$ corresponds to a certain relation on the set of isomorphism classes of simple right $A$-modules defined in purely categorical terms. In case of an arbitrary left module category $\mathcal{M}$ over a finite tensor category $\mathcal{C}$ such a relation was introduced by Etingof and Ostrik [12, Lemma 3.8]. It was proved there that this relation is symmetric under the assumption that $\mathcal{C}$ has projective covers and $P \otimes X$ is projective in $\mathcal{M}$ for any projective object $P \in \mathcal{C}$ and any object $X \in \mathcal{M}$. The second condition is rather nontrivial to verify.

Lemma 4.8. Suppose that $A$ has semilocal localizations with respect to a central subring $Z$. Given $P, Q \in \operatorname{Max} A$ with $P \leq_{H} Q$, let $\mathfrak{n}=Q \cap Z$. If either (a) $A_{\mathfrak{n}} / \mathfrak{n} A_{\mathfrak{n}}$ is semiprimary or (b) the antipode of $H$ is bijective, then there exists $Q^{\prime} \in \operatorname{Max}_{\mathfrak{n}} A$ satisfying $P \leq_{H} Q^{\prime}$ and $Q^{\prime} \leq_{H} P$.

Proof. Recall from Lemma 2.1 that both rings $A / P$ and $A / Q$ are simple artinian. Let $V$ and $W$ be as in Lemma 4.7. Then $W$ is a subfactor of the right $A$-module $M=\operatorname{Hom}(U, V)$ for some finite dimensional $U \in \mathcal{M}^{H}$. Denoting by $\rho: U \rightarrow U \otimes H$ the comodule structure map, we have $\rho(U) \subset U \otimes C$ for some finite dimensional subcoalgebra $C \subset H$. Since $V P=0$, it is immediate from the definition of the $A$ module structure on $M$ that $P_{C}$ annihilates $M$. Put $\mathfrak{a}=P_{C} \cap Z$. The commutative $\operatorname{ring} Z / \mathfrak{a}$ is semiprimary by Lemma 3.6 ; it is therefore a finite direct product of primary rings. Then $A / \mathfrak{a} A \cong \prod_{\mathfrak{m} \in X} A_{\mathfrak{m}} / \mathfrak{a} A_{\mathfrak{m}}$ where $X$ is the finite set of those $\mathfrak{m} \in \operatorname{Max} Z$ for which $\mathfrak{a} \subset \mathfrak{m}$. Since $M \mathfrak{a}=0$, we have $M \cong \prod_{\mathfrak{m} \in X} M_{\mathfrak{m}}$, and $A$ operates in $M_{\mathfrak{m}}$ via the projection onto $A_{\mathfrak{m}} / \mathfrak{a} A_{\mathfrak{m}}$. Then $W$ is a subfactor of $M_{\mathfrak{m}}$ for some $\mathfrak{m} \in X$. Since $W \mathfrak{n}=0$, while all elements of $Z \backslash \mathfrak{m}$ are invertible on $W$, we must have $\mathfrak{n} \subset \mathfrak{m}$. As $\mathfrak{n} \in \operatorname{Max} Z$ by Lemma 2.1, this yields $\mathfrak{n}=\mathfrak{m}$, showing that $\mathfrak{n} \in X$ and $M_{\mathfrak{n}} \neq 0$.

Suppose that (a) holds. The primary $\operatorname{ring} Z_{\mathfrak{n}} / \mathfrak{a} Z_{\mathfrak{n}}$ has a nilpotent maximal ideal generated by $\mathfrak{n}$. Hence $\mathfrak{n} A_{\mathfrak{n}} / \mathfrak{a} A_{\mathfrak{n}}$ is a nilpotent ideal of $A_{\mathfrak{n}} / \mathfrak{a} A_{\mathfrak{n}}$, and it follows that $A_{\mathfrak{n}} / \mathfrak{a} A_{\mathfrak{n}}$ is semiprimary. Since $M_{\mathfrak{n}}$ is a nonzero right $A_{\mathfrak{n}} / \mathfrak{a} A_{\mathfrak{n}}$-module, it contains a simple submodule, say $W^{\prime}$. Denote by $Q^{\prime}$ the annihilator of $W^{\prime}$ in $A$. As $W^{\prime}$ is a simple submodule of $M$, Lemma 4.7(ii) yields $P \leq_{H} Q^{\prime}$ and $Q^{\prime} \leq_{H} P$. Since $W^{\prime} \mathfrak{n}=0$, we get $Q^{\prime} \cap Z=\mathfrak{n}$.

Suppose now that (b) holds. Then $U \cong\left(U^{\prime}\right)^{*}$ for some $U^{\prime} \in \mathcal{M}^{H}$, $\operatorname{dim} U^{\prime}<\infty$. By Lemma 4.6 $M \cong U^{\prime} \otimes V$, so $M$ is finitely generated in $\mathcal{M}_{A}$ according to [29, Lemma 1.1]. The direct summand $M_{\mathfrak{n}}$ of $M$ is also finitely generated in $\mathcal{M}_{A}$. Then $M_{\mathfrak{n}}$ has a simple factor module, call it $W^{\prime}$. We now complete the proof similarly to 
case (a), but using Lemma 4.7(iii) instead.

We say that $A$ has quasilocal localizations with respect to a central subring $Z$ if $A_{\mathfrak{m}}$ is quasilocal and $\mathfrak{m} A_{\mathfrak{m}} \subset \operatorname{Jac}\left(A_{\mathfrak{m}}\right)$ for each $\mathfrak{m} \in \operatorname{Max} Z$.

Proposition 4.9. Suppose that $A$ has quasilocal localizations with respect to $Z$. If either all rings $A_{\mathfrak{m}} / \mathfrak{m} A_{\mathfrak{m}}, \mathfrak{m} \in \operatorname{Max} Z$, are primary or the antipode of $H$ is bijective then the relation $\leq_{H}$ is symmetric on $\operatorname{Max} A$.

Proof. Since $A_{\mathfrak{n}}$ is quasilocal for any $\mathfrak{n} \in \operatorname{Max} Z$, there is a single ideal in $\operatorname{Max}_{\mathfrak{n}} A$. Hence $Q^{\prime}=Q$ in the notation of Lemma 4.8.

Corollary 4.10. If $A$ is commutative then $\leq_{H}$ is symmetric on Max $A$.

Proof. The hypotheses of Proposition 4.9 are satisfied if we take $Z=A$.

\section{Projectivity result for module algebras}

Let $A$ be an $H$-module algebra and $M \in{ }_{H} \mathcal{M}_{A}$. The compatibility of the two module structures on $M$ is expressed as

$$
h(v a)=\sum\left(h_{(1)} v\right)\left(h_{(2)} a\right) \quad \text { for } h \in H, v \in M, a \in A .
$$

Lemma 5.1. Suppose that $C$ is a subcoalgebra of $H$ and $I$ an ideal of $A$ such that $A / I$ is weakly finite. If $M / M I \cong(A / I)^{n}$ in $\mathcal{M}_{A}$ and the A-module $M / M I_{C}$ is $n$-generated, then $M / M I_{C} \cong\left(A / I_{C}\right)^{n}$ in $\mathcal{M}_{A}$.

Proof. We will regard $\operatorname{Hom}(C, M / M I)$ as a right $\operatorname{Hom}(C, A / I)$-module by means of the convolution action. If $\xi: C \rightarrow A / I$ and $\eta: C \rightarrow M / M I$ are linear maps, then

$$
(\eta \xi)(c)=\sum \eta\left(c_{(1)}\right) \xi\left(c_{(2)}\right), \quad c \in C .
$$

For each ideal $J$ of $A$ denote by $\pi_{J}: M \rightarrow M / M J$ the canonical projection. Define $\hat{m}, \tilde{m} \in \operatorname{Hom}(C, M / M I)$ for each $m \in M$ by the rules

$$
\hat{m}(c)=\varepsilon(c) \pi_{I}(m), \quad \tilde{m}(c)=\pi_{I}(\mathrm{~cm})
$$

Pick $e_{1}, \ldots, e_{n} \in M$ such that $\pi_{I}\left(e_{1}\right), \ldots, \pi_{I}\left(e_{n}\right)$ are a basis for the $A / I$-module $M / M I$. Given $\eta \in \operatorname{Hom}(C, M / M I)$, there are uniquely determined $\xi_{1}, \ldots, \xi_{n} \in$ $\operatorname{Hom}(C, A / I)$ such that $\eta(c)=\sum_{i=1}^{n} \pi_{I}\left(e_{i}\right) \xi_{i}(c)$ for all $c \in C$, which is equivalent to $\eta=\sum_{i=1}^{n} \hat{e}_{i} \xi_{i}$. Hence $\hat{e}_{1}, \ldots, \hat{e}_{n}$ are a basis for the $\operatorname{Hom}(C, A / I)$-module $\operatorname{Hom}(C, M / M I)$.

Pick any elements $v_{1}, \ldots, v_{n}$ generating $M$ modulo $M I_{C}$. Given $m \in M$, there exist $\zeta_{1}, \ldots, \zeta_{n} \in \operatorname{Hom}(H, A)$ such that

$$
S(h) m \equiv \sum_{i=1}^{n} v_{i} \zeta_{i}(h)\left(\bmod M I_{C}\right)
$$

for all $h \in H$. Note that $C\left(M I_{C}\right) \subset M I=\operatorname{Ker} \pi_{I}$. Taking $c \in C$, we get

$$
\begin{aligned}
\varepsilon(c) m=\sum_{(c)} c_{(1)} S\left(c_{(2)}\right) m & \equiv \sum_{(c)} c_{(1)}\left(\sum_{i=1}^{n} v_{i} \zeta_{i}\left(c_{(2)}\right)\right) \\
& \equiv \sum_{i=1}^{n} \sum_{(c)}\left(c_{(1)} v_{i}\right)\left(c_{(2)} \zeta_{i}\left(c_{(3)}\right)\right)(\bmod M I),
\end{aligned}
$$


and applying $\pi_{I}$, we deduce $\hat{m}(c)=\sum_{i=1}^{n} \sum_{(c)} \tilde{v}_{i}\left(c_{(1)}\right) \theta_{i}\left(c_{(2)}\right)$ where the $\theta_{i}$ 's are linear maps $C \rightarrow A / I$ defined by the formula $\theta_{i}(c)=\sum_{(c)} c_{(1)} \zeta_{i}\left(c_{(2)}\right)+I$. This shows that $\hat{m}=\sum_{i=1}^{n} \tilde{v}_{i} \theta_{i}$. In particular, the submodule of $\operatorname{Hom}(C, M / M I)$ generated by $\tilde{v}_{1}, \ldots, \tilde{v}_{n}$ contains $\hat{e}_{1}, \ldots, \hat{e}_{n}$. So $\tilde{v}_{1}, \ldots, \tilde{v}_{n}$ generate the whole $\operatorname{Hom}(C, M / M I)$. Since the algebra $\operatorname{Hom}(C, A / I)$ is weakly finite $\left[28\right.$, Lemma 7.1], $\tilde{v}_{1}, \ldots, \tilde{v}_{n}$ are in fact a basis for $\operatorname{Hom}(C, M / M I)$ over $\operatorname{Hom}(C, A / I)$.

Suppose that $x_{1}, \ldots, x_{n} \in A$ are any elements such that $\sum_{i=1}^{n} v_{i} x_{i} \in M I_{C}$. Then

$$
\sum_{i=1}^{n} \sum_{(c)}\left(c_{(1)} v_{i}\right)\left(c_{(2)} x_{i}\right)=c\left(\sum_{i=1}^{n} v_{i} x_{i}\right) \in C\left(M I_{C}\right) \subset M I
$$

for all $c \in C$. Applying $\pi_{I}$, we rewrite this as $\sum_{i=1}^{n} \tilde{v}_{i} \tilde{x}_{i}=0$ where $\tilde{x}_{i} \in \operatorname{Hom}(C, A / I)$ is defined as in section 3, i.e., $\tilde{x}_{i}(c)=c x_{i}+I$ for $c \in C$. We must have $\tilde{x}_{i}=0$, i.e., $x_{i} \in I_{C}$ for each $i=1, \ldots, n$. Hence $\pi_{I_{C}}\left(v_{1}\right), \ldots, \pi_{I_{C}}\left(v_{n}\right)$ are linearly independent over $A / I_{C}$.

Further on we assume that $A$ has semilocal localizations with respect to a central subring $Z$. For a nonnegative $r \in \mathbb{Q}$ and a finitely generated right $A$-module $M$ the open subsets $U_{r}(M) \subset \operatorname{Max} Z$ and the ideals $I_{r}(M)$ of $A$ were defined in section 1. When $M \in{ }_{H} \mathcal{M}_{A}$, we use the same notation ignoring the $H$-module structure. Denote by $J_{r}(M)$ the smallest $H$-stable ideal of $A$ containing $I_{r}(M)$.

Lemma 5.2. Let $r=r_{P}(M)$ where $P \in \operatorname{Max} A$ and $M \in{ }_{H} \mathcal{M}_{A}$ is an A-finite object. Suppose that $r_{Q}(M) \leq r$ for each $Q \in \operatorname{Max} A$ such that $P \leq_{H} Q$. Let $r=n / l$ for some integers $l>0, n \geq 0$. Then:

(i) $r_{Q}(M)=r$ for each $Q \in \operatorname{Max} A$ with $P \leq_{H} Q$.

(ii) $\left(M / M P_{C}\right)^{l} \cong\left(A / P_{C}\right)^{n}$ in $\mathcal{M}_{A}$ for subcoalgebras $C$ of $H$ with $\operatorname{dim} C<\infty$.

Proof. The ring $A / P$ is simple artinian by Lemma 2.1. Then $(M / M P)^{l} \cong(A / P)^{n}$ in $\mathcal{M}_{A}$ since the two $A / P$-modules here have equal lengths. Let $C$ be given as in (ii). According to Lemma $3.6 A / P_{C}$ is a semilocal ring. Since $r_{Q}(M) l \leq r l=n$ for any $Q \in \operatorname{Max} A$ with $P_{C} \subset Q$, it follows from Lemma 1.6(i) that the $A / P_{C^{-}}$ module $\left(M / M P_{C}\right)^{l}$ is $n$-generated. Now Lemma 5.1 applied to $M^{l} \in{ }_{H} \mathcal{M}_{A}$ yields the isomorphism in (ii). Then $(M / M Q)^{l} \cong(A / Q)^{n}$, and so $r_{Q}(M)=r$, for any $Q \in \operatorname{Max} A$ with $P_{C} \subset Q$. As this holds for all finite dimensional subcoalgebras $C$, we deduce (i).

Lemma 5.3. Let $r=r_{P}(M)$ where $P \in \operatorname{Max} A$ and $M \in{ }_{H} \mathcal{M}_{A}$ is an A-finite object. Suppose that $U_{r}(M)$ is quasicompact and $r_{Q}(M) \leq r$ for each $Q \in \operatorname{Max} A$ such that $Q \cap Z=Q^{\prime} \cap Z$ for some $Q^{\prime} \in \operatorname{Max} A$ with $P \leq_{H} Q^{\prime}$. Then $J_{r}(M) \subset P$.

Proof. The isomorphism in Lemma 5.2(ii) enables us to apply Lemma 2.5(ii) with $R=A$ and $K=P_{C}$. We conclude that $I_{r}(M) \subset P_{C}$ for each finite dimensional subcoalgebra $C$ of $H$, whence $I_{r}(M) \subset P_{H}$. Since $P_{H}$ is an $H$-stable ideal of $A$, it follows that $J_{r}(M) \subset P_{H} \subset P$.

Recall from Lemma 2.4 that $r(M)=\sup \left\{r_{P}(M) \mid P \in \operatorname{Max} A\right\}$.

Proposition 5.4. Given any A-finite object $M \in{ }_{H} \mathcal{M}_{A}$ and $P \in \operatorname{Max} A$ one has $r_{P}(M)=r(M)$ if and only if $P \supset J_{r(M)}(M)$. Moreover, $J_{r(M)}(M) \neq A$. 
Proof. Let $r=r(M)$. We have $r_{Q}(M) \leq r$ for all $Q \in \operatorname{Max} A$. So by Lemma 2.3 $U_{r}(M)=\operatorname{Max} Z$, which is a quasicompact space. If $r_{P}(M)=r$ then $P$ satisfies the hypothesis of Lemma 5.3, whence $J_{r}(M) \subset P$. Conversely, if $J_{r}(M) \subset P$, then also $I_{r}(M) \subset P$, whence $r_{P}(M)=r$ by Lemma 2.5(iii) (where we take $R=A, K=P$ ). Lemma 2.4 says that $r_{P}(M)=r$ for at least one $P \in \operatorname{Max} A$. Hence $J_{r}(M) \neq A$.

Corollary 5.5. Suppose that $M \in{ }_{H} \mathcal{M}_{A}$ is an A-finite object and $A$ has a maximal ideal $P$ such that $r_{P}(M)=r(M)$ and $P$ contains no nonzero $H$-stable ideals of $A$. Let $r(M)=n / l$ for some integers $n \geq 0, l>0$. Then:

(i) $r_{Q}(M)=r(M)$ for all $Q \in \operatorname{Max} A$.

(ii) $M$ is projective in $\mathcal{M}_{A} ; M$ is a generator in $\mathcal{M}_{A}$ provided $M \neq 0$.

(iii) $M_{\mathfrak{p}}^{l} \cong A_{\mathfrak{p}}^{n}$ in $\mathcal{M}_{A_{\mathfrak{p}}}$ for each $\mathfrak{p} \in \operatorname{Spec} Z$.

Proof. Let $r=r(M)$. Since $J_{r}(M)$ is an $H$-stable ideal of $A$ contained in $P$, we get $J_{r}(M)=0$; so $I_{r}(M)=0$ too. Then $J_{r}(M) \subset Q$ for any $Q \in \operatorname{Max} A$, whence (i) holds by Proposition 5.4. Since $U_{r}(M)=\operatorname{Max} Z$, we have $M_{\mathfrak{m}}^{l} \cong A_{\mathfrak{m}}^{n}$ for any $\mathfrak{m} \in \operatorname{Max} Z$ by Proposition 1.11(ii). If $\mathfrak{p} \in \operatorname{Spec} Z$, then $\mathfrak{p} \subset \mathfrak{m}$ for some $\mathfrak{m} \in \operatorname{Max} Z$. Since $A_{\mathfrak{p}}$ is a localization of $A_{\mathfrak{m}}$, we have $M_{\mathfrak{p}} \cong M_{\mathfrak{m}} \otimes_{A_{\mathfrak{m}}} A_{\mathfrak{p}}$, whence (iii). Note that $M$ is projective or a generator in $\mathcal{M}_{A}$ if and only if so is $M^{l}$. Hence Lemma 2.6 applied to $M^{l}$ establishes (ii).

Theorem 5.6. Suppose that $A$ is an $H$-simple $H$-module algebra which has semilocal localizations with respect to a central subring $Z$. Let $M$ be any locally A-finite object of ${ }_{H} \mathcal{M}_{A}$. Put $l=\operatorname{gcd}\{$ length $A / Q \mid Q \in \operatorname{Max} A\}$. Then:

(i) $M$ is projective in $\mathcal{M}_{A} ; M$ is a generator in $\mathcal{M}_{A}$ provided $M \neq 0$.

(ii) $M_{\mathfrak{p}}^{l}$ is a free $A_{\mathfrak{p}}$-module for each $\mathfrak{p} \in \operatorname{Spec} Z$.

(iii) If $M$ is not $A$-finite then $M_{\mathfrak{p}}$ is a free $A_{\mathfrak{p}}$-module for each $\mathfrak{p} \in \operatorname{Spec} Z$.

Proof. If $M$ is $A$-finite, then there exists $P \in \operatorname{Max} A$ with $r_{P}(M)=r(M)$ by Lemma 2.4. We may now apply Corollary 5.5. For each $Q \in \operatorname{Max} A$ we have $r(M)=r_{Q}(M)$, whence $r(M) \cdot \operatorname{length}(A / Q) \in \mathbb{Z}$. It follows that $r(M) l \in \mathbb{Z}$, and so Corollary 5.5 establishes both (i) and (ii).

Suppose further that $M$ is not $A$-finite. The family $\mathcal{F}$ of all ${ }_{H} \mathcal{M}_{A}$-subobjects of $M$ clearly satisfies condition (a) of Lemma 5.7 below. If $N \in \mathcal{F}$ and $N \neq M$ then, since $M$ is locally $A$-finite, there exists a nonzero $A$-finite subobject $F \subset M$ such that $F \not \subset N$. We have $N^{\prime}=N+F \in \mathcal{F}$ and $N$ is properly contained in $N^{\prime}$. Furthermore, $N^{\prime} / N \cong F /(F \cap N)$ is an $A$-finite object of ${ }_{H} \mathcal{M}_{A}$; as we have proved already, $N^{\prime} / N$ is projective in $\mathcal{M}_{A}$. Thus condition (b) of Lemma 5.7 is also fulfilled, and (i) follows.

Let $\mathfrak{m} \in \operatorname{Max} Z$. If $N \in \mathcal{F}$ and $N \neq M$ then $M / N$ is a generator in $\mathcal{M}_{A}$ by (i). In this case $(M / N)_{\mathfrak{m}}$ is a generator in $\mathcal{M}_{A_{\mathfrak{m}}}$, whence $N_{\mathfrak{m}} \neq M_{\mathfrak{m}}$. In particular, this holds for any $A$-finite subobject of $M$ since $M$ is not $A$-finite. As a consequence, the $A_{\mathfrak{m}}$-module $M_{\mathfrak{m}}$ cannot be finitely generated. The freeness of $M_{\mathfrak{m}}$ now follows from Lemma 5.8 which we apply by considering the family of submodules $N_{\mathfrak{m}}$ of $M_{\mathfrak{m}}$ with $N \in \mathcal{F}$. If $\mathfrak{p} \in \operatorname{Spec} Z$, then $M_{\mathfrak{p}} \cong M_{\mathfrak{m}} \otimes_{A_{\mathfrak{m}}} A_{\mathfrak{p}}$ for any $\mathfrak{m} \in \operatorname{Max} Z$ containing $\mathfrak{p}$. This proves (iii).

Lemma 5.7. Let $R$ be any ring. A right $R$-module $M$ has to be projective provided that there exists a family $\mathcal{F}$ of submodules of $M$ satisfying 
(a) $\{0\} \in \mathcal{F}$ and the union of every chain in $\mathcal{F}$ is again in $\mathcal{F}$,

(b) each $N \in \mathcal{F}, N \neq M$, is properly contained in some $N^{\prime} \in \mathcal{F}$ such that $N^{\prime} / N$ is projective in $\mathcal{M}_{R}$.

If at least one $N \in \mathcal{F}$ is a generator of $\mathcal{M}_{R}$ then $M$ is a generator too.

Proof. Let $\xi: V \rightarrow W$ be any epimorphism and $\varphi: M \rightarrow W$ any morphism in $\mathcal{M}_{R}$. By Zorn's Lemma there exist a maximal element in the set $X$ of all pairs $(N, \psi)$ where $N \in \mathcal{F}$ and $\psi: N \rightarrow V$ is an $\mathcal{M}_{R}$-morphism such that $\xi \circ \psi=\left.\varphi\right|_{N}$. If $N$, $N^{\prime}$ are as in (b), then $N^{\prime}=N \oplus G$ for some projective submodule $G$; it is then clear that any $\psi$ occurring as a component of $(N, \psi) \in X$ can be extended to an $\mathcal{M}_{R}$-morphism $\psi^{\prime}: N^{\prime} \rightarrow V$ with the property that $\left(N^{\prime}, \psi^{\prime}\right) \in X$. Therefore every maximal element of $X$ has to be $(M, \psi)$ where $\psi: M \rightarrow V$ is an $\mathcal{M}_{R^{-} \text {-morphism }}$ satisfying $\xi \circ \psi=\varphi$. This proves that $M$ is projective. Moreover, the $R$-module $M / N$ is projective for each $N \in \mathcal{F}$ since the family of submodules $N^{\prime} / N$ with $N^{\prime} \in \mathcal{F}$ and $N^{\prime} \supset N$ satisfies (a) and (b). Hence each $N \in \mathcal{F}$ is a direct summand of $M$, and the final assertion of the lemma is clear.

Lemma 5.8. Let $R$ be a semilocal ring. A right $R$-module $M$ is necessarily free as long as $M$ is not finitely generated and there is a family $\mathcal{F}$ of submodules satisfying

(a) $\{0\} \in \mathcal{F}$ and the union of every chain in $\mathcal{F}$ is again in $\mathcal{F}$,

(b) each $N \in \mathcal{F}, N \neq M$, is properly contained in some $N^{\prime} \in \mathcal{F}$ such that $\left(N^{\prime} / N\right)^{l}$ is a finitely generated free $R$-module for some $l \in \mathbb{Z}_{+}$.

This is a restatement of [28, Lemma 2.5].

Lemma 5.9. Let $M \in{ }_{H} \mathcal{M}_{A}$ be an $A$-finite object. Suppose that $M \neq 0$ and $A$ is not $H$-simple. Then $A$ has a nonzero $H$-stable ideal $I$ such that $M I \neq M$.

Proof. Suppose that $M I=M$ for each nonzero $H$-stable ideal $I$ of $A$. Since $M \neq 0$, we have $r(M)>0$. If $P$ is any maximal ideal of $A$ for which $r_{P}(M)=r(M)$, then $M P \neq M$, and therefore $P$ cannot contain nonzero $H$-stable ideals of $A$. Now Corollary 5.5 shows that $M$ is a generator in $\mathcal{M}_{A}$. Then $M I \neq M$ for each proper ideal $I$ of $A$. It follows that $A$ cannot have $H$-stable ideals other than 0 and $A$, i.e. $A$ is $H$-simple.

\section{Local projectivity and flatness}

Here we consider an $H$-module algebra $A$ which is not $H$-simple, but there is a prime ideal of $A$ containing no nonzero $H$-stable ideals. We want to look at the localizations $M_{\mathfrak{p}}$ at a single prime of $Z$. In contrast to Theorem 5.6 we are able to prove the projectivity of $M_{\mathfrak{p}}$ only under additional restrictions.

Proposition 6.1. Let $A$ be an $H$-module algebra which has semilocal localizations with respect to $Z$. Suppose that $\operatorname{Max} Z$ is noetherian and either all rings $A_{\mathfrak{m}} / \mathfrak{m} A_{\mathfrak{m}}$, $\mathfrak{m} \in \operatorname{Max} Z$, are semiprimary or the antipode of $H$ is bijective. Let $M \in{ }_{H} \mathcal{M}_{A}$ be an $A$-finite object whose rank function $Q \mapsto r_{Q}(M)$ is constant on each fibre $\operatorname{Max}_{\mathfrak{m}} A$, $\mathfrak{m} \in \operatorname{Max} Z$. Let $r \in \mathbb{Q}$ and $P \in \operatorname{Max} A$. Then:

(i) $r_{P}(M)=r$ if and only if $P \supset J_{r}(M)$ and $P \not \supset J_{s}(M)$ for any $s>r$.

(ii) $r_{Q}(M)=r_{P}(M)$ for each $Q \in \operatorname{Max} A$ satisfying $P \leq_{H} Q$.

Assuming that $P$ contains no nonzero $H$-stable ideals of $A$ and $r_{P}(M)=n / l$ for some integers $n \geq 0, l>0$, we also have: 
(iii) $r_{Q}(M) \geq r_{P}(M)$ for all $Q \in \operatorname{Max} A$.

(iv) $M_{\mathfrak{n}}^{l} \cong A_{\mathfrak{n}}^{n}$ in $\mathcal{M}_{A_{\mathfrak{n}}}$ for any $\mathfrak{n} \in \operatorname{Max} Z$ such that $r_{Q}(M)=r_{P}(M)$ on $\operatorname{Max}_{\mathfrak{n}} A$.

Proof. Since Max $Z$ is noetherian, for any real $x>0$ the open subset $\bigcup_{s<x} U_{s}(M)$ is quasicompact. Hence there exists $t \in \mathbb{Q}, t<x$, such that $U_{s}(M)=U_{t}(M)$ for each $s \in \mathbb{Q}$ satisfying $t<s<x$. Given $Q \in \operatorname{Max} A$ and $\mathfrak{n}=Q \cap Z$, we have, by Lemma 2.3, $\mathfrak{n} \in U_{s}(M)$ if and only if $r_{Q}(M) \leq s$ since the rank function of $M$ is constant on $\operatorname{Max}_{\mathfrak{n}} A$. It follows that $r_{Q}(M) \leq t$ whenever $r_{Q}(M)<x$.

The previous argument shows that for any subset $X \subset \operatorname{Max} A$ there exists $P^{\prime} \in$ $X$ such that $r_{Q}(M) \leq r_{P^{\prime}}(M)$ for all $Q \in X$. For, if we let $x=\sup \left\{r_{Q}(M) \mid Q \in X\right\}$ and take $P^{\prime}$ with $r_{P^{\prime}}(M)$ sufficiently close to $x$, we must have $r_{P^{\prime}}(M)=x$.

Now choose $P^{\prime}$ as above in the subset $X=\left\{Q \in \operatorname{Max} A \mid P \leq_{H} Q\right\}$. Denote $x=r_{P^{\prime}}(M)$. We have $P \leq_{H} P^{\prime}$. If $Q \in \operatorname{Max} A$ satisfies $P^{\prime} \leq_{H} Q$, then also $P \leq_{H} Q$, i.e., $Q \in X$. By the assumption on the rank function of $M$ we get $r_{Q^{\prime}}(M)=r_{Q}(M) \leq x$ for any $Q^{\prime} \in \operatorname{Max} A$ with $Q^{\prime} \cap Z=Q \cap Z$. Thus $P^{\prime}$ satisfies the hypotheses of Lemmas 5.2, 5.3. We deduce that $J_{x}(M) \subset P^{\prime}$ and $r_{Q}(M)=x$ for any $Q \in \operatorname{Max} A$ with $P^{\prime} \leq_{H} Q$.

By Lemma 4.8 there exists $P^{\prime \prime} \in \operatorname{Max} A$ such that $P^{\prime \prime} \cap Z=P^{\prime} \cap Z$, while both $P \leq_{H} P^{\prime \prime}$ and $P^{\prime \prime} \leq_{H} P$ hold. The first condition on $P^{\prime \prime}$ shows that $r_{P^{\prime \prime}}(M)=x$, while the second condition gives $P^{\prime \prime} \in X$. But then we may replace $P^{\prime}$ with $P^{\prime \prime}$ and conclude that $r_{Q}(M)=x$ for any $Q \in \operatorname{Max} A$ with $P^{\prime \prime} \leq_{H} Q$. In particular, $r_{P}(M)=x$. Now we may replace $P^{\prime}$ with $P$. The earlier conclusions about $P^{\prime}$ yield (ii) and verify the inclusion $J_{x}(M) \subset P$.

If $s \in \mathbb{Q}$ is such that $r_{P}(M)<s$ then $r_{Q}(M)<s$ for all $Q \in \operatorname{Max}_{\mathfrak{m}} A$ where $\mathfrak{m}=P \cap Z$. Lemma 2.5(iii) applied with $R=A, K=P$ shows that $I_{s}(M) \not \subset P$; then also $J_{s}(M) \not \subset P$ for such $s$. But we have checked already that $J_{s}(M) \subset P$ for $s=r_{P}(M)$. The last two statements are equivalent to (i).

Suppose that $P$ contains no nonzero $H$-stable ideals of $A$. Then $J_{x}(M)=0$. By (i) applied to an arbitrary $Q \in \operatorname{Max} A$, the inclusion $J_{x}(M) \subset Q$ yields $r_{Q}(M) \geq x$, proving (iii). If $\mathfrak{n}$ is as in (iv), then $\mathfrak{n} \in U_{x}(M)$. Since $I_{x}(M)=0$, Lemma 1.11(ii) verifies (iv).

Proposition 6.2. Let $A$ be an $H$-module algebra, module-finite over a central subring $Z$ such that $Z$ is a Jacobson ring with a noetherian space Max $Z$. Suppose that $P \in \operatorname{Spec} A$ contains no nonzero $H$-stable ideals of $A$. Let $M \in{ }_{H} \mathcal{M}_{A}$ be an $A$-finite object whose rank function $Q \mapsto r_{Q}(M)$ is constant on each fibre $\operatorname{Max}_{\mathfrak{m}} A$, $\mathfrak{m} \in \operatorname{Max} Z$. Put $m=\inf \left\{r_{Q}(M) \mid Q \in \operatorname{Max} A\right\}$, and let $m=n / l$ for some integers $n \geq 0, l>0$. Then there exists $z \in Z, z \notin P$, such that:

(i) $r_{Q}(M)=m$ for each $Q \in \operatorname{Max} A$ with $z \notin Q$.

(ii) $M_{z}^{l} \cong A_{z}^{n}$ in $\mathcal{M}_{A_{z}}$; hence $M_{\mathfrak{q}}^{l} \cong A_{\mathfrak{q}}^{n}$ in $\mathcal{M}_{A_{\mathfrak{q}}}$ for each $\mathfrak{q} \in \operatorname{Spec} Z$ with $z \notin \mathfrak{q}$.

Proof. Let $\mathfrak{p}=P \cap Z$; clearly $\mathfrak{p} \in \operatorname{Spec} Z$. As we pointed out in the Remark at the end of section $2, A_{\mathfrak{p}}$ is semilocal. For $r \in \mathbb{Q}$ we have $\mathfrak{p} \in \widetilde{U}_{r}(M)$ if and only if $r_{Q^{\prime}}\left(M_{\mathfrak{p}}\right) \leq r$ for all $Q^{\prime} \in \operatorname{Max} A_{\mathfrak{p}}$ (this follows from Lemma 1.6 and the definition of $\left.\widetilde{U}_{r}(M)\right)$. Hence there exists the smallest $r$ with the previous property, namely $r=\max \left\{r_{Q^{\prime}}\left(M_{\mathfrak{p}}\right) \mid Q^{\prime} \in \operatorname{Max} A_{\mathfrak{p}}\right\}$. We will assume that $r$ is this number.

Since $\widetilde{U}_{r}(M)$ is an open neighborhood of $\mathfrak{p}$ in $\operatorname{Spec} Z$, there exists a basic open subset $D(z) \subset \widetilde{U}_{r}(M)$ for some $z \in Z \backslash \mathfrak{p}$. We choose such a $z$. If $Q$ is a maximal 
ideal of $A$ with $z \notin Q$, then $Q \in \operatorname{Max}_{\mathfrak{n}} A$ where $\mathfrak{n} \in D(z) \cap \operatorname{Max} Z \subset U_{r}(M)$, so that $r_{Q}(M) \leq r$.

By Lemma 2.8 there exists a subset $X \subset \operatorname{Max} A$ such that $P=\bigcap_{Q \in X} Q$ and $z \notin Q$ for each $Q \in X$. Suppose that $Q \in X$ and $\mathfrak{n}=Q \cap Z$. Since $P \subset Q$, we have $\mathfrak{p} \subset \mathfrak{n}$. If $s=r_{Q}(M)$, then $\mathfrak{n} \in U_{s}(M)$, and so $\widetilde{U}_{s}(M)$ is an open neighbourhood of $\mathfrak{n}$ in $\operatorname{Spec} Z$. It follows that $\mathfrak{p} \in \widetilde{U}_{s}(M)$, which yields $s \geq r$ by the choice of $r$. Since the opposite inequality has been established, we conclude that $r_{Q}(M)=r$. Now $J_{r}(M) \subset Q$ by Proposition 6.1(i).

It follows that $J_{r}(M) \subset P$. Since $P$ contains no nonzero $H$-stable ideals, we get $J_{r}(M)=0$. Then $I_{r}(M)=0$ too. Thus $J_{r}(M) \subset Q$ for any $Q \in \operatorname{Max} A$; Proposition 6.1 (i) ensures that $r_{Q}(M) \geq r$. Note that $U_{r}(M) \neq \varnothing$ since $X \neq \varnothing$. It follows that $m=r$, and the previous inequalities prove (i).

Lemma 1.11(ii) shows that $M_{\mathfrak{n}}^{l} \cong A_{\mathfrak{n}}^{n}$ when $\mathfrak{n} \in U_{r}(M)$. Since $Z$ is a Jacobson ring and its localization $Z_{z}$ at $z$ is a finitely generated $Z$-algebra, every maximal ideal of $Z_{z}$ contracts to a maximal ideal of $Z[4, \mathrm{Ch} . \mathrm{V}, \S 3, \mathrm{Th} .3]$. Thus the maximal ideals of $Z_{z}$ are of the form $\mathfrak{n} Z_{z}$ with $\mathfrak{n} \in \operatorname{Max} Z, z \notin \mathfrak{n}$. We know that $\mathfrak{n} \in U_{r}(M)$ for any such $\mathfrak{n}$. It follows that $A_{z}$ has semilocal localizations with respect to $Z_{z}$ and the right $A_{z}$-modules $M_{z}^{l}$ and $A_{z}^{n}$ have isomorphic localizations at all maximal ideals of $Z_{z}$. Thus we may apply Lemma 2.6 to the $A_{z}$-module $M_{z}^{l}$. Replacing $z$ with a suitable element $z^{\prime}$ such that $Z_{z^{\prime}}$ is a localization of $Z_{z}$, we prove (ii).

A restriction on the rank function is a serious deficiency of Propositions 6.1, 6.2. This restriction is void in the case where all sets $\operatorname{Max}_{\mathfrak{m}} A$ are singletons.

Theorem 6.3. Let $A$ be an $H$-module algebra, module-finite over a central subring $Z$ such that $Z$ is a Jacobson ring with a noetherian space $\operatorname{Max} Z$ and each maximal ideal of $Z$ is contained in a single maximal ideal of $A$. Suppose that $P \in \operatorname{Spec} A$ contains no nonzero $H$-stable ideals of $A$. Denote $\mathfrak{p}=P \cap Z$ and

$$
l=\operatorname{gcd}\left\{\text { length } A_{\mathfrak{p}} / Q^{\prime} \mid Q^{\prime} \in \operatorname{Max} A_{\mathfrak{p}}\right\} .
$$

Then $M_{\mathfrak{p}}^{l}$ is a free $A_{\mathfrak{p}}$-module for any locally $A$-finite $M \in{ }_{H} \mathcal{M}_{A}$.

Proof. When $M$ is $A$-finite, we may apply Proposition 6.2. The $A_{\mathfrak{p}}$-module $M_{\mathfrak{p}}^{l^{\prime}}$ is free for some $l^{\prime} \in \mathbb{Z}_{+}$. Hence $r=r_{Q^{\prime}}\left(M_{\mathfrak{p}}\right)$ does not depend on $Q^{\prime} \in \operatorname{Max} A_{\mathfrak{p}}$. Since $A_{\mathfrak{p}}$ is semilocal, $M_{\mathfrak{p}}^{l^{\prime}}$ is free in $\mathcal{M}_{A_{\mathfrak{p}}}$ for any $l^{\prime} \in \mathbb{Z}_{+}$such that $r l^{\prime} \in \mathbb{Z}$. Since $r \cdot$ length $A_{\mathfrak{p}} / Q^{\prime} \in \mathbb{Z}$ for any $Q^{\prime} \in \operatorname{Max} A_{\mathfrak{p}}$, we have $r l \in \mathbb{Z}$, whence the conclusion.

Suppose that $M$ is not $A$-finite. If $N, N^{\prime}$ are any two ${ }_{H} \mathcal{M}_{A}$-subobjects of $M$ such that $N^{\prime} / N$ is $A$-finite then $\left(N_{\mathfrak{p}}^{\prime} / N_{\mathfrak{p}}\right)^{l}$ is a free $A_{\mathfrak{p}}$-module. If the $A_{\mathfrak{p}}$-module $M_{\mathfrak{p}}$ is finitely generated, then $M_{\mathfrak{p}}=N_{\mathfrak{p}}$ for some $A$-finite subobject, and the conclusion is clear. Otherwise we apply Lemma 5.8 by considering the family of submodules $N_{\mathfrak{p}}$ of $M_{\mathfrak{p}}$ with $N$ running through all ${ }_{H} \mathcal{M}_{A}$-subobjects of $M$.

Theorem 6.4. Let $B$ be any $H$-module algebra, $A$ an $H$-stable subalgebra contained in the center of $B$. Suppose that $A$ is a Jacobson ring with a noetherian space Max $A$ and $I B=B$ for each nonzero $H$-stable ideal $I$ of $A$. Then each locally $A$-finite object $M \in{ }_{H} \mathcal{M}_{B}$ is flat in $\mathcal{M}_{A}$.

Proof. Given a monomorphism $\varphi: V \rightarrow W$ in $\mathcal{M}_{A}$, denote by $K$ the kernel of the map id $\otimes \varphi: M \otimes_{A} V \rightarrow M \otimes_{A} W$. Since the latter map may be regarded as an $\mathcal{M}_{B}$-morphism, $K$ is a $B$-module. Suppose that $x \in K$ is a nonzero element. Denote 
by $\mathfrak{a}$ the annihilator of $x$ in $A$. Then $x$ is annihilated by the ideal $\mathfrak{a} B$ of $B$, and therefore $\mathfrak{a} B \neq B$. There exists $Q \in \operatorname{Max} B$ such that $\mathfrak{a} B \subset Q$. Now $\mathfrak{p}=Q \cap A$ is a prime ideal of $A$ and $\mathfrak{a} \subset \mathfrak{p}$. Since $\mathfrak{p} B \neq B$, none of the nonzero $H$-stable ideals of $A$ can be contained in $\mathfrak{p}$. Since $A$ is commutative, we may apply Theorem 6.3 with $Z=A$ and $P=\mathfrak{p}$. We deduce that $M_{\mathfrak{p}}$ is projective in $\mathcal{M}_{A_{\mathfrak{p}}}$, which implies that the map

$$
\text { id } \otimes \varphi \otimes \text { id }: M \otimes_{A} V \otimes_{A} A_{\mathfrak{p}} \rightarrow M \otimes_{A} W \otimes_{A} A_{\mathfrak{p}}
$$

is injective. On the other hand, the kernel of this map coincides with $K \otimes_{A} A_{\mathfrak{p}}$ since $A_{\mathfrak{p}}$ is flat in $\mathcal{M}_{A}$. Thus $K \otimes_{A} A_{\mathfrak{p}}=0$. Then $x$ is annihilated by an element in $Z \backslash \mathfrak{p}$, i.e. $\mathfrak{a} \not \subset \mathfrak{p}$. This contradiction shows that $K=0$.

\section{Dualization to comodule algebras}

Let $H$ be a bialgebra and $A$ a right $H$-comodule algebra. An object of $\mathcal{M}_{A}^{H}$ will be called $A$-finite if it is finitely generated in $\mathcal{M}_{A}$. An arbitrary object $M$ is a directed union of its $A$-finite subobjects. Indeed, any finite subset of $M$ is contained in a finite dimensional $H$-subcomodule; the $A$-submodule generated by the latter is an $A$-finite subobject.

Lemma 7.1. Each object of $\mathcal{M}_{A}^{H}$ is flat (resp. projective) in $\mathcal{M}_{A}$ provided that this is true for all A-finite objects. Each nonzero object of $\mathcal{M}_{A}^{H}$ is a projective generator in $\mathcal{M}_{A}$ provided that this is true for all nonzero A-finite objects.

Proof. Since tensor products commute with filtered direct limits, the flat part of the lemma follows from the fact that each $M \in \mathcal{M}_{A}^{H}$ is a directed union of $A$-finite subobjects. The projective part follows from Lemma 5.7 in which we take $\mathcal{F}$ to be the family of all subobjects of $M$.

Let $H^{\prime}$ be a second bialgebra, $A^{\prime}$ an $H^{\prime}$-comodule algebra. Given a homomorphism of bialgebras $\varphi: H^{\prime} \rightarrow H$, we may view $A^{\prime}$ as an $H$-comodule algebra. Suppose that we are given also a map $A^{\prime} \rightarrow A$ which is a homomorphism of $H$-comodule algebras. In this case there is a functor $\mathcal{M}_{A^{\prime}}^{H^{\prime}} \rightsquigarrow \mathcal{M}_{A}^{H}$ which takes an object $N \in \mathcal{M}_{A^{\prime}}^{H^{\prime}}$ to $N \otimes_{A^{\prime}} A \in \mathcal{M}_{A}^{H}$ on which the comodule structure $N \otimes_{A^{\prime}} A \rightarrow\left(N \otimes_{A^{\prime}} A\right) \otimes H$ is given by the rule

$$
v \otimes a \mapsto \sum\left(v_{(0)} \otimes a_{(0)}\right) \otimes \varphi\left(v_{(1)}\right) a_{(1)}
$$

where $v \in N, a \in A$. It is easy to check that this map is well-defined. In the special case where $H^{\prime}=H$ and $A^{\prime}=k$ with the trivial comodule structure, we obtain an object $V \otimes A \in \mathcal{M}_{A}^{H}$ for each right $H$-comodule $V$.

We next make several observations concerning direct limits of comodule algebras. Suppose that $H=\lim _{\longrightarrow} H_{i}$, the direct limit of an inductive family $\mathcal{H}=\left(H_{i}\right)$ of bialgebras indexed by a directed set $\mathcal{I}$. An $\mathcal{H}$-compatible inductive family of comodule algebras $\mathcal{F}=\left(A_{i}\right)$ is a collection containing for each $i \in \mathcal{I}$ an $H_{i}$-comodule algebra $A_{i}$ and for each pair $i, j \in \mathcal{I}$ with $i \leq j$ a homomorphism of $H_{j}$-comodule algebras $A_{i} \rightarrow A_{j}$; these maps are requested to obey the usual rules of inductive systems. If such an $\mathcal{F}$ is given, $A=\underline{\lim } A_{i}$ becomes an $H$-comodule algebra in a natural way. We mention below several properties of the category $\mathcal{M}_{A}^{H}$ under previous assumptions.

We say that $M \in \mathcal{M}_{A}^{H}$ is $\mathcal{F}$-induced if there exists $i \in \mathcal{I}$ and $N \in \mathcal{M}_{A_{i}}^{H_{i}}$ such that $M \cong N \otimes_{A_{i}} A$. Denote by $\mathcal{F}_{A}^{H}$ the class of all $A$-finite objects of $\mathcal{M}_{A}^{H}$ isomorphic to $N \otimes A_{i} A$ for some $i \in \mathcal{I}$ and an $A_{i}$-finite $N \in \mathcal{M}_{A_{i}}^{H_{i}}$. 
Lemma 7.2. If $M \in \mathcal{M}_{A}^{H}$ is an $\mathcal{F}$-induced object and $M^{\prime}$ any $A$-finite subobject, then $M / M^{\prime}$ is $\mathcal{F}$-induced. In this case $M / M^{\prime} \in \mathcal{F}_{A}^{H}$ whenever $M \in \mathcal{F}_{A}^{H}$.

Proof. Let $M \cong N \otimes_{A_{i}} A$ for some $i \in \mathcal{I}$ and $N \in \mathcal{M}_{A_{i}}^{H}$. Put $J=\{j \in \mathcal{I} \mid i \leq j\}$. For each $j \in J$ denote $N_{j}=N \otimes_{A_{i}} A_{j}$, and let $M_{j}$ be the image of the canonical map $\varphi_{j}: N_{j} \rightarrow M$. We thus obtain a directed family of vector subspaces of $M$ indexed by $J$. Since $A$ is covered by the images of $A_{j}, j \in J$, we have $M=\bigcup_{j \in J} M_{j}$. By the hypothesis $M^{\prime}$ is generated in $\mathcal{M}_{A}$ by a finite subset. The latter is contained in a finite dimensional $H$-subcomodule $V \subset M^{\prime}$, and we then have $M^{\prime}=V A$. There exists $j \in J$ such that $V \subset M_{j}$. We may view $\varphi_{j}$ as an $\mathcal{M}^{H}$-morphism. Hence there exists a finite dimensional $H$-subcomodule $W$ of $N_{j}$ such that $\varphi_{j}(W)=V$. Let $\rho_{j}: N_{j} \rightarrow N_{j} \otimes H_{j}$ be the $H_{j}$-comodule structure map. We must have

$$
\rho_{j}(W) \subset W \otimes H_{j}+N_{j} \otimes \operatorname{Ker}\left(H_{j} \rightarrow H\right) .
$$

Then $\rho_{j}(W) \subset W \otimes H_{j}+N_{j} \otimes U$ for some finite dimensional subspace in the kernel of $H_{j} \rightarrow H$. Now $U$ vanishes in $H_{t}$ for some $t \in J, t \geq j$. If we denote by $W^{\prime}$ the image of $W$ in $N_{t}$, then $W^{\prime}$ is an $H_{t}$-subcomodule of $N_{t}$ satisfying $\varphi_{t}\left(W^{\prime}\right)=V$. The map $W^{\prime} \otimes A_{t} \rightarrow N_{t}$ afforded by the $A_{t}$-module structure is a morphism in $\mathcal{M}_{A_{t}}^{H_{t}}$; hence its cokernel $K$ is an object of that category. Tensoring with $A$, we obtain an exact sequence

$$
\left(W^{\prime} \otimes A_{t}\right) \otimes_{A_{t}} A \rightarrow N_{t} \otimes_{A_{t}} A \cong M \rightarrow K \otimes_{A_{t}} A \rightarrow 0
$$

in $\mathcal{M}_{A}^{H}$. By construction the image of the first map coincides with $M^{\prime}$. It follows that $M / M^{\prime} \cong K \otimes_{A_{t}} A$ is an $\mathcal{F}$-induced object. Note that $K$ is $A_{t}$-finite whenever $N$ is $A_{i}$-finite.

Lemma 7.3. Every A-finite object $M \in \mathcal{M}_{A}^{H}$ is isomorphic to a factor object of an object from $\mathcal{F}_{A}^{H}$.

Proof. There exists a finite dimensional $H$-subcomodule $V \subset M$ such that $M=V A$. The map $V \otimes A \rightarrow M$ afforded by the $A$-module structure is then an epimorphism in $\mathcal{M}_{A}^{H}$. So it remains to prove the conclusion of the lemma for the object $V \otimes A$.

Since $H$ is an injective cogenerator in $\mathcal{M}^{H}$, we can embed $V$ as a subcomodule in $H^{n}$ for some integer $n>0$. For each $i \in \mathcal{I}$ let $\varphi_{i}: H_{i}^{n} \rightarrow H^{n}$ denote the canonical map. Then $V$ is contained in the image of $\varphi_{j}$ for some $j \in \mathcal{I}$. Since $\varphi_{j}$ may be regarded as an $\mathcal{M}^{H}$-morphism, there exists a finite dimensional $H$-subcomodule $W \subset H_{j}^{n}$ such that $\varphi_{j}(W)=V$. As in the proof of previous lemma we can find $t \in \mathcal{I}, t \geq j$, such that the image $W^{\prime}$ of $W$ in $H_{t}^{n}$ is an $H_{t}$-subcomodule. The map $W^{\prime} \rightarrow V$ obtained by restriction of $\varphi_{t}$ is an epimorphism in $\mathcal{M}^{H}$; it gives rise to an epimorphism $W^{\prime} \otimes A \rightarrow V \otimes A$ in $\mathcal{M}_{A}^{H}$. Since $W^{\prime} \otimes A \cong\left(W^{\prime} \otimes A_{t}\right) \otimes A_{t} A$ and $W^{\prime} \otimes A_{t}$ is an $A_{t}$-finite object of $\mathcal{M}_{A_{t}}^{H_{t}}$, we have $W^{\prime} \otimes A \in \mathcal{F}_{A}^{H}$.

Lemma 7.4. Suppose $A$ has a homomorphism into a nonzero artinian ring $R$ and each nonzero object from $\mathcal{F}_{A}^{H}$ is a projective generator in $\mathcal{M}_{A}$. Then $\mathcal{F}_{A}^{H}$ contains all A-finite objects of $\mathcal{M}_{A}^{H}$.

Proof. For each $N \in \mathcal{F}_{A}^{H}$ the $R$-module $N \otimes_{A} R$ is finitely generated, and we denote by $l(N)$ its length. If $N \neq 0$, then $N$ is a projective generator in $\mathcal{M}_{A}$; in this case $N \otimes_{A} R$ is a projective generator in $\mathcal{M}_{R}$, whence $N \otimes_{A} R \neq 0$, i.e. $l(N)>0$. 
If $M \in \mathcal{M}_{A}^{H}$ is $A$-finite, Lemma 7.3 ensures the existence of an $\mathcal{M}_{A}^{H}$-epimorphism $\varphi: F \rightarrow M$ with $F \in \mathcal{F}_{A}^{H}$. If $M$ is projective in $\mathcal{M}_{A}$, then $\varphi$ splits in $\mathcal{M}_{A}$, and therefore $\operatorname{Ker} \varphi$ is an $A$-finite object of $\mathcal{M}_{A}^{H}$. In this case $M \in \mathcal{F}_{A}^{H}$ by Lemma 7.2.

Suppose now that $M \in \mathcal{F}_{A}^{H}$ and $M^{\prime}$ is any $A$-finite $\mathcal{M}_{A}^{H}$-subobject of $M$. By Lemma 7.2 $M / M^{\prime} \in \mathcal{F}_{A}^{H}$. By the hypothesis $M$ and $M / M^{\prime}$ are both projective in $\mathcal{M}_{A}$. Then so is $M^{\prime}$ too. This implies $M^{\prime} \in \mathcal{F}_{A}^{H}$ as we have observed above. Since the exact sequence $0 \rightarrow M^{\prime} \rightarrow M \rightarrow M / M^{\prime} \rightarrow 0$ splits in $\mathcal{M}_{A}$, it remains exact after tensoring with $R$, whence $l(M)=l\left(M^{\prime}\right)+l\left(M / M^{\prime}\right)$. If $M^{\prime} \neq M$ then $l\left(M / M^{\prime}\right)>0$, in which case $l\left(M^{\prime}\right)<l(M)$.

Given another $A$-finite subobject $M^{\prime \prime}$ of $M$ properly containing $M^{\prime}$, we have then $l\left(M^{\prime}\right)<l\left(M^{\prime \prime}\right)$ since $M^{\prime \prime}$ is also in $\mathcal{F}_{A}^{H}$. It is now clear that $M$ satisfies ACC on $A$-finite subobjects. But every subobject of $M$ is a directed union of $A$-finite ones; hence it is itself $A$-finite. So, according to Lemma 7.2, the class $\mathcal{F}_{A}^{H}$ is closed under factor objects, and we are done.

For the ring $R$ appearing in the next lemma we say that a finitely generated projective $R$-module $G$ has constant rank if $r_{P}(G)$, as defined in section 1, does not depend on $P \in \operatorname{Max} R$; we denote by $r(G)$ this common value. If $G \neq 0$, then $G$ has a simple factor module annihilated by some $P$, and therefore $r(G)=r_{P}(G)>0$. If $G \cong G^{\prime} \oplus G^{\prime \prime}$ in $\mathcal{M}_{R}$, then $r_{P}(G)=r_{P}\left(G^{\prime}\right)+r_{P}\left(G^{\prime \prime}\right)$ for all $P$; hence $G^{\prime \prime}$ has constant rank whenever so do both $G$ and $G^{\prime}$. In this case $r\left(G^{\prime}\right)<r(G)$ unless $G^{\prime \prime}=0$. There are only finitely many possible values of $r\left(G^{\prime}\right)$ when $G^{\prime}$ runs through the direct summands of $G$ having constant rank; indeed, $r\left(G^{\prime}\right)<r(G)$ and $r\left(G^{\prime}\right) l \in \mathbb{Z}$ for any $G^{\prime}$ where $l$ is the greatest common divisor of the lengths of the simple artinian factor rings of $R$. It follows that $G$ satisfies ACC on direct summands of constant rank.

Lemma 7.5. Let $A \rightarrow R$ be a homomorphism into a ring $R$ all whose right primitive factor rings are artinian. Suppose that for each $F \in \mathcal{F}_{A}^{H}$ the $R$-module $F \otimes_{A} R$ is projective of constant rank. Then for each $A$-finite $N \in \mathcal{M}_{A}^{H}$ there exists an epimorphism $\xi: F \rightarrow N$ in $\mathcal{M}_{A}^{H}$ such that $F \in \mathcal{F}_{A}^{H}$ and the map $F \otimes_{A} R \rightarrow N \otimes_{A} R$ induced by $\xi$ is an isomorphism in $\mathcal{M}_{R}$.

Proof. Let $M \in \mathcal{F}_{A}^{H}$. By the hypothesis $M \otimes_{A} R$ is a projective $R$-module of constant rank. For each $\mathcal{M}_{A}^{H}$-subobject $K \subset M$ denote by $T_{K}$ the image of the canonical map $K \otimes_{A} R \rightarrow M \otimes_{A} R$. If $M^{\prime} \subset M$ is an $A$-finite subobject, then $M / M^{\prime} \in \mathcal{F}_{A}^{H}$ by Lemma 7.2. In this case $M / M^{\prime} \otimes_{A} R$ is a projective $R$-module of constant rank, and it follows from the exact sequence

$$
M^{\prime} \otimes_{A} R \rightarrow M \otimes_{A} R \rightarrow M / M^{\prime} \otimes_{A} R \rightarrow 0
$$

that so too is $T_{M^{\prime}}$. As a consequence, $M \otimes_{A} R$ satisfies ACC on submodules of the form $T_{M^{\prime}}$ with $M^{\prime}$ as above. An arbitrary subobject $K \subset M$ is a directed union of $A$-finite ones. Then $T_{K}=\bigcup T_{K^{\prime}}$ where $K^{\prime}$ runs through the $A$-finite subobjects of $K$, and it follows that $T_{K}=T_{K^{\prime}}$ for some $K^{\prime}$ of this type. Then the canonical projection $\xi: M / K^{\prime} \rightarrow M / K$ induces an isomorphism after tensoring with $R$. Thus for $N=M / K$ we have the desired conclusion with $F=M / K^{\prime}$. Lemma 7.3 completes the proof.

Proposition 7.6. Let $H=\lim _{\longrightarrow} H_{i}$ and $A=\lim _{\longrightarrow} A_{i}$ as before. 
(i) All objects of $\mathcal{M}_{A}^{H}$ are flat in $\mathcal{M}_{A}$ provided that for each $i$ all objects of $\mathcal{M}_{A_{i}}^{H_{i}}$ are flat in $\mathcal{M}_{A_{i}}$.

(ii) Suppose that $A$ has a homomorphism into a nonzero artinian ring $R$. If for each $i$ all nonzero objects of $\mathcal{M}_{A_{i}}^{H_{i}}$ are projective generators in $\mathcal{M}_{A_{i}}$ then all nonzero objects of $\mathcal{M}_{A}^{H}$ are projective generators in $\mathcal{M}_{A}$.

(iii) Let $A \rightarrow R$ be a ring homomorphism where $R$ is a ring all whose right primitive factor rings are artinian. Suppose that for each $i$ and each $A_{i}$-finite $N \in \mathcal{M}_{A_{i}}^{H_{i}}$ the $R$-module $N \otimes_{A_{i}} R$ is projective of constant rank. Then $M \otimes_{A} R$ is projective in $\mathcal{M}_{R}$ for any $M \in \mathcal{M}_{A}^{H}$; if $M$ is $A$-finite then $M \otimes_{A} R$ has constant rank.

Proof. (i) Since $N \otimes_{A_{i}} A$ is flat in $\mathcal{M}_{A}$ whenever $N$ is flat in $\mathcal{M}_{A_{i}}$, the hypothesis implies that every $\mathcal{F}$-induced object of $\mathcal{M}_{A}^{H}$ is flat in $\mathcal{M}_{A}$. An arbitrary $A$-finite object $L \in \mathcal{M}_{A}^{H}$ is isomorphic to $M / K$ where $M \in \mathcal{F}_{A}^{H}$ and $K$ is an $\mathcal{M}_{A}^{H}$-subobject of $M$. We have $L \cong \lim M / K^{\prime}$ where $K^{\prime}$ ranges over all $A$-finite subobjects of $K$. By Lemma 7.2 $M / K^{\prime} \in \mathcal{F}_{A}^{H}$ for each $K^{\prime}$. Thus $L$ is a direct limit of flat $A$-modules; then $L$ is flat in $\mathcal{M}_{A}$. Lemma 7.1 completes the proof.

(ii) The hypothesis implies that all nonzero $\mathcal{F}$-induced objects of $\mathcal{M}_{A}^{H}$ are projective generators in $\mathcal{M}_{A}$. Lemma 7.4 shows that the same conclusion holds for all $A$-finite objects, and Lemma 7.1 establishes this for arbitrary objects.

(iii) Here $M \otimes_{A} R$ is a projective $R$-module of constant rank for each $M \in \mathcal{F}_{A}^{H}$. By Lemma 7.5 the same holds for each $A$-finite object of $\mathcal{M}_{A}^{H}$. In order to extend this to arbitrary objects we have to repeat the proof of [28, Th. 1.2] given in case of commutative algebras. The proof is easier under the assumption that $R$ is flat in ${ }_{A} \mathcal{M}$. In this case we can apply Lemma 5.7 to the family of submodules $N \otimes_{A} R$ of $M \otimes_{A} R$ where $N$ runs through all $\mathcal{M}_{A}^{H}$-subobjects of $M$.

Recall that the finite dual $H^{\circ}$ of $H$ is a subalgebra of $H^{*}$ consisting of all linear functions vanishing on an ideal of finite codimension in $H$. There is a comultiplication on $H^{\circ}$ dual to the multiplication on $H$. Moreover, $H^{\circ}$ is a Hopf algebra whenever so is $H$. As explained in [30], $\mathcal{M}^{H}$ is equivalent to the category of rational left $H^{*}$-modules. This gives a functor $\mathcal{M}^{H} \rightsquigarrow H^{\circ} \mathcal{M}$. If $A$ is a right $H$-comodule algebra, then $A$ is a left $H^{\circ}$-module algebra with respect to the corresponding module structure; then we obtain a functor $\mathcal{M}_{A}^{H} \rightsquigarrow H^{\circ} \mathcal{M}_{A}$. Moreover, all objects in the image of that functor are locally $A$-finite.

If $H$ is residually finite dimensional, then $H^{\circ}$ is dense in $H^{*}$; it follows that the subcomodules of any $U \in \mathcal{M}^{H}$ coincide with the submodules of the corresponding $H^{\circ}$-module. In this case the $H$-costable ideals of an $H$-comodule algebra $A$ coincide with the $H^{\circ}$-stable ideals, and $A$ is an $H$-simple $H$-comodule algebra if and only if $A$ is an $H^{\circ}$-simple $H^{\circ}$-module algebra.

It is easy now to translate the results from the preceding sections to the context of comodule algebras. The next result is the comodule version of Theorem 5.6.

Theorem 7.7. Let $M \in \mathcal{M}_{A}^{H}$ where $H$ is a residually finite dimensional Hopf algebra and $A$ is an $H$-simple $H$-comodule algebra which has semilocal localizations with respect to a central subring $Z$. Put $l=\operatorname{gcd}\{\operatorname{length} A / Q \mid Q \in \operatorname{Max} A\}$. Then:

(i) $M$ is projective in $\mathcal{M}_{A} ; M$ is a generator in $\mathcal{M}_{A}$ provided $M \neq 0$.

(ii) $M_{\mathfrak{p}}^{l}$ is a free $A_{\mathfrak{p}}$-module for each $\mathfrak{p} \in \operatorname{Spec} Z$.

(iii) If $M$ is not $A$-finite then $M_{\mathfrak{p}}$ is a free $A_{\mathfrak{p}}$-module for each $\mathfrak{p} \in \operatorname{Spec} Z$. 
In case of commutative comodule algebras we can weaken the assumption about the Hopf algebra $H$.

Theorem 7.8. Suppose $H$ is a directed union of residually finite dimensional Hopf subalgebras and $A$ is a commutative $H$-comodule algebra. If $\mathfrak{p} \in \operatorname{Spec} A$ contains no nonzero $H$-costable ideals of $A$ then $M_{\mathfrak{p}}$ is a free $A_{\mathfrak{p}}$-module for any $M \in \mathcal{M}_{A}^{H}$.

Proof. If $H$ is residually finite dimensional and $A$ is finitely generated, the conclusion follows from Theorem 6.3 since in this case $Z=A$ is a noetherian Jacobson ring. In general there is a directed family $\mathcal{G}$ of residually finite dimensional Hopf subalgebras of $H$ whose union coincides with $H$. Let $\mathcal{I}$ be the set of all pairs $\left(A^{\prime}, H^{\prime}\right)$ where $H^{\prime} \in \mathcal{G}$ and $A^{\prime}$ is a finitely generated subalgebra of $A$ such that $\rho_{A}\left(A^{\prime}\right) \subset A^{\prime} \otimes H^{\prime}$. Given two pairs $\left(A_{1}, H_{1}\right)$ and $\left(A_{2}, H_{2}\right)$ from $\mathcal{I}$, there exists $H_{3} \in \mathcal{G}$ containing both $H_{1}$ and $H_{2}$; clearly $\left(A_{1} A_{2}, H_{3}\right) \in \mathcal{I}$. This shows that $\mathcal{I}$ is directed by inclusion.

For $\left(A^{\prime}, H^{\prime}\right) \in \mathcal{I}$ we may regard $A^{\prime}$ as an $H^{\prime}$-comodule algebra. Any $H^{\prime}$-costable ideal of $A^{\prime}$ extends to an $H$-costable ideal of $A$. It follows then that the prime ideal $\mathfrak{p}^{\prime}=\mathfrak{p} \cap A^{\prime}$ of $A^{\prime}$ contains no nonzero $H^{\prime}$-costable ideals of $A^{\prime}$. Hence $N \otimes_{A^{\prime}} A_{\mathfrak{p}^{\prime}}^{\prime}$ is a free $A_{\mathfrak{p}^{\prime}}^{\prime}$-module for any $N \in \mathcal{M}_{A^{\prime}}^{H^{\prime}}$; since the homomorphism $A^{\prime} \rightarrow A_{\mathfrak{p}}$ factors through $A_{\mathfrak{p}^{\prime}}^{\prime}$, the $A_{\mathfrak{p}}$-module $N \otimes_{A^{\prime}} A_{\mathfrak{p}}$ is also free. The assignment $\left(A^{\prime}, H^{\prime}\right) \mapsto H^{\prime}$ defines an inductive family $\mathcal{H}$ of Hopf algebras indexed by $\mathcal{I}$. The direct limit of $\mathcal{H}$ is equal to $H$. The assignment $\left(A^{\prime}, H^{\prime}\right) \mapsto A^{\prime}$ defines an $\mathcal{H}$-compatible inductive family $\mathcal{F}$ of comodule algebras. If $V$ is any $H$-subcomodule of $A$ with $\operatorname{dim} V<\infty$, then $\rho_{A}(V) \subset V \otimes H^{\prime}$ for some $H^{\prime} \in \mathcal{G}$; denoting by $A^{\prime}$ the subalgebra of $A$ generated by $V$, we have $\left(A^{\prime}, H^{\prime}\right) \in \mathcal{I}$. Since $A$ is covered by its finite dimensional subcomodules, the direct limit of $\mathcal{F}$ equals $A$.

It remains to apply Proposition 7.6(iii) with $R=A_{\mathfrak{p}}$. Since the ring $A_{\mathfrak{p}}$ is local, all projective $A_{\mathfrak{p}}$-modules are free by Kaplansky's Theorem.

Theorem 7.8 implies the next result whose proof follows that of Theorem 6.4.

Theorem 7.9. Let $H$ be a directed union of residually finite dimensional Hopf subalgebras, $B$ an $H$-comodule algebra, and $A$ an $H$-costable subalgebra contained in the center of $B$. Suppose that $I B=B$ for each nonzero $H$-costable ideal $I$ of $A$. Then each object $M \in \mathcal{M}_{B}^{H}$ is flat in $\mathcal{M}_{A}$.

In conclusion we will deduce all results stated in the introduction. The structure theorem for objects of $\mathcal{M}_{H}^{H}[30$, Th. 4.1.1] shows that any Hopf algebra $H$ is a simple object of $\mathcal{M}_{H}^{H}$. If $A$ is a Hopf subalgebra of $H$, then $A$ is simple in $\mathcal{M}_{A}^{A}$, and therefore simple in $\mathcal{M}_{A}^{H}$. In this case $A$ is an $H$-simple $H$-comodule algebra.

If $A$ is a right coideal subalgebra of $H$, then $I H=H$ for each $H$-costable ideal $I \neq 0$ of $A$ since $I H$ is an $\mathcal{M}_{H}^{H}$-subobject of $H$. Furthermore, the opposite multiplication makes $A^{\mathrm{op}}$ into a right coideal subalgebra of the bialgebra $H^{\mathrm{op}}$. If the antipode of $H$ is bijective, $H^{\mathrm{op}}$ is actually a Hopf algebra. The previous argument applied to $A^{\mathrm{op}}, H^{\mathrm{op}}$ yields $H I=H$ for each nonzero $H$-costable ideal $I$ of $A$.

Theorem 7.10. Let $H$ be a residually finite dimensional Hopf algebra, $A$ be a Hopf subalgebra having semilocal localizations with respect to a central subring $Z$. Then each nonzero object $M \in \mathcal{M}_{A}^{H}$ is a projective generator in $\mathcal{M}_{A}$ and $M_{\mathfrak{p}}$ is a free $A_{\mathfrak{p}}$-module for any $\mathfrak{p} \in \operatorname{Spec} Z$.

Proof. We apply Theorem 7.7 in which $l=1$ since $k$ is a factor algebra of $A$. 
In particular, $H$ is a projective generator in $\mathcal{M}_{A}$. We may change both the multiplication and comultiplication in $A$ and $H$ to the opposite ones, obtaining another pair of Hopf algebras $A^{\mathrm{op}, \mathrm{cop}} \subset H^{\mathrm{op} \text {,cop }}$. Theorem 7.10 applied to the latter shows that $H$ is a projective generator in ${ }_{A} \mathcal{M}$. Thus Theorem 0.1 is proved.

Theorem 7.11. Let $A \subset B \subset H$ where $H$ is a residually finite dimensional Hopf algebra, $B$ is a Hopf subalgebra, and $A$ is a right coideal subalgebra which has semilocal localizations with respect to a central subring $Z$. Suppose that $B$ is right modulefinite over $A$ and the antipode of $B$ is bijective. Then each nonzero object $M \in \mathcal{M}_{A}^{H}$ is a projective generator in $\mathcal{M}_{A}$ and $M_{\mathfrak{p}}$ is a free $A_{\mathfrak{p}}$-module for any $\mathfrak{p} \in \operatorname{Spec} Z$.

Proof. We have $B I=B$ for each nonzero $H$-costable ideal $I$ of $A$. Hence we may apply Lemma 5.9 regarding $M=B$ as an $A$-finite object of $H^{\circ} \mathcal{M}_{A}$. It follows that $A$ is an $H$-simple $H$-comodule algebra. Again Theorem 7.7 applies.

Theorem 7.12. Let $A \subset B \subset H$ where $H$ is a directed union of residually finite dimensional Hopf subalgebras, $B$ is any Hopf subalgebra, and $A$ is a right coideal subalgebra contained in the center of $B$. Then:

(i) $M_{\mathfrak{p}}$ is a free $A_{\mathfrak{p}}$-module for each $M \in \mathcal{M}_{A}^{H}$ and $\mathfrak{p} \in \operatorname{Spec} A$ with $\mathfrak{p} B \neq B$.

(ii) Each object of $\mathcal{M}_{B}^{H}$ is flat in $\mathcal{M}_{A}$.

Proof. Since $\Delta(A) \subset(A \otimes H) \cap(B \otimes B)=A \otimes B$, we may regard $A$ as a right coideal subalgebra of $B$. Furthermore, an ideal $I$ of $A$ is $H$-costable if and only if it is $B$-costable. It follows that $I B=B$ for each nonzero $H$-costable ideal $I$ of $A$. In particular, $\mathfrak{p} \in \operatorname{Spec} A$ cannot contain nonzero $H$-costable ideals of $A$ whenever $\mathfrak{p} B \neq B$. The two conclusions are therefore consequences of Theorems 7.8, 7.9.

Theorem 7.13. Let $A$ be a commutative Hopf subalgebra of a Hopf algebra $H$ which is a directed union of residually finite dimensional Hopf subalgebras. Then each nonzero object of $\mathcal{M}_{A}^{H}$ is a projective generator in $\mathcal{M}_{A}$.

Proof. If $H$ is residually finite dimensional then the conclusion is a special case of Theorem 7.10. In general we apply this to each residually finite dimensional Hopf subalgebra $H^{\prime}$ of $H$ and the right coideal subalgebra $A \cap H^{\prime}$ of $H^{\prime}$. Proposition 7.6(ii) completes the proof.

Remark. If $A$ is contained in the center of $H$ then Theorem 7.13 can be proved by first observing that $H$ is faithfully flat in $\mathcal{M}_{A}$ and then using [32, Th. 5].

\section{References}

1. G. Azumaya, On maximally central algebras, Nagoya Math. J. 2 (1951) 119-150.

2. H. Bass, Algebraic K-theory, Benjamin, 1968.

3. J.-E. Björk, Noetherian and Artinian chain conditions of associative rings, Arch. Math. 24 (1973) 366-378.

4. N. Bourbaki, Commutative Algebra, Springer, 1989.

5. K.A. Brown and K.R. Goodearl, Lectures on Algebraic Quantum Groups, Birkhäuser, 2002 .

6. R. Camps and W. Dicks, On semilocal rings, Isr. J. Math. 81 (1993) 203-211.

7. W. Chin, Spectra of smash products, Isr. J. Math. 72 (1990) 84-98.

8. B. Cortzen and L.W. Small, Finite extensions of rings, Proc. Amer. Math. Soc. 103 (1988) 1058-1062.

9. C.W. Curtis, Noncommutative extensions of Hilbert rings, Proc. Amer. Math. Soc. 4 (1953) 945-955. 
10. C. De Concini and V. Lyubashenko, Quantum function algebra at roots of 1 , Adv. Math. 108 (1994) 205-262.

11. Y. Doi, On the structure of relative Hopf modules, Comm. Algebra 11 (1983) 243-255.

12. P. Etingof and V. Ostrik, Finite tensor categories, Moscow Math. J. 4 (2004) 627-654.

13. K. Hoffmann, Coidealunteralgebren in endlich dimensionalen Hopfalgebren, Dissertation, Univ. München, 1991.

14. V.K. Kharchenko, PBW-bases of coideal subalgebras and a freeness theorem, Preprint.

15. T.-Y. Lam, A First Course in Noncommutative Rings, Graduate Texts in Mathematics, Vol. 131, Springer, 1991.

16. A. Masuoka, On Hopf algebras with cocommutative coradicals, J. Algebra 144 (1991) 451-466.

17. A. Masuoka, Freeness of Hopf algebras over coideal subalgebras, Comm. Algebra 20 (1992) 1353-1373.

18. A. Masuoka and D. Wigner, Faithful flatness of Hopf algebras, J. Algebra 170 (1994) $156-164$.

19. S. Montgomery, Hopf algebras and Their Actions on Rings, CBMS Regional Conference Series in Mathematics, Vol. 82, American Mathematical Society, 1993.

20. S. Montgomery and H.-J. Schneider, Hopf crossed products, rings of quotients, and prime ideals, Adv. Math. 112 (1995) 1-55.

21. S. Montgomery and H.-J. Schneider, Prime ideals in Hopf Galois extensions, Isr. J. Math. 112 (1999) 187-235.

22. W.D. Nichols and M.B. Zoeller, A Hopf algebra freeness theorem, Amer. J. Math. 111 (1989) 381-385.

23. C. Procesi, Non commutative Jacobson-rings, Ann. Scuola Norm. Sup. Pisa 21 (1967) 281-290.

24. D.E. Radford, Pointed Hopf algebras are free over Hopf subalgebras, J. Algebra 45 (1977) 266-273.

25. J.C. Robson and L.W. Small, Liberal extensions, Proc. London Math. Soc. 42 (1981) 87-103.

26. P. Schauenburg, Faithful flatness over Hopf subalgebras: counterexamples, in "Interactions between ring theory and representations of algebras", Lect. Notes Pure Appl. Math., Vol. 210, Marcel Dekker, 2000, pp. 331-344.

27. H.-J. Schneider, Some remarks on exact sequences of quantum groups, Comm. Algebra 21 (1993) 3337-3357.

28. S. Skryabin, Projectivity and freeness over comodule algebras, to appear in Trans. Amer. Math. Soc.

29. S. Skryabin and F. Van Oystaeyen, The Goldie Theorem for H-semiprime algebras, J. Algebra 305 (2006) 292-320.

30. M.E. Sweedler, Hopf Algebras, Benjamin, 1969.

31. M. Takeuchi, Free Hopf algebras generated by coalgebras, J. Math. Soc. Japan 23 (1971) 561-582.

32. M. Takeuchi, Relative Hopf modules-equivalences and freeness criteria, J. Algebra 60 (1979) 452-471.

33. Q.-S. Wu and J.J. Zhang, Noetherian PI Hopf algebras are Gorenstein, Trans. Amer. Math. Soc. 355 (2003) 1043-1066. 